\title{
On the transition from the Ginzburg-Landau equation to the extended Fisher-Kolmogorov equation
}

\author{
Vivi Rottschäfer and Arjen Doelman \\ Mathematisch Instituut \\ Universiteit Utrecht \\ Postbus 80.010 \\ 3508TA Utrecht \\ The Netherlands
}

\begin{abstract}
The Ginzburg-Landau (GL) equation 'generically' describes the behaviour of small perturbations of a marginally unstable basic state in systems on unbounded domains. In this paper we consider the transition from this generic situation to a degenerate (co-dimension 2) case in which the GL approach is no longer valid. Instead of studying a general underlying model problem, we consider a two-dimensional system of coupled reaction-diffusion equations in one spatial dimension. We show that near the degeneration the behaviour of small perturbations is governed by the extended Fisher-Kolmogorov (eFK) equation (at leading order). The relation between the GL-equation and the eFK-equation is quite subtle, but can be analysed in detail. The main goal of this paper is to study this relation, which we do asymptotically. The asymptotic analysis is compared to numerical simulations of the full reaction-diffusion system. As one approaches the co-dimension 2 point, we observe that the stable stationary periodic patterns predicted by the GL-equation evolve towards various different families of stable, stationary (but not necessarily periodic) so-called 'multi-bump' solutions. In the literature, these multi-bump patterns are shown to exist as solutions of the eFK equation, but there is no proof of the asymptotic stability of these solutions. Our results suggest that these multi-bump patterns can also be asymptotically stable in large classes of model problems.
\end{abstract}




\section{Introduction}

The behaviour near criticality of small solutions of a system of partial differential equations on an unbounded domain can be described by so-called modulation or amplitude equations. The most well-known, and 'generic' modulation equation is the (complex) Ginzburg-Landau (GL) equation

$$
A_{\tau}=r A+c \Delta A+h A|A|^{2},
$$

where $A$ is a complex valued function, $r$ a (real) control or bifurcation parameter and $c$ and $h$ complex parameters which are determined by the underlying 'full' system of PDE's (see for instance [14]). Generally, $h$ is referred to as the Landan-constant.

The derivation of the GL-equation is based on a detailed analysis of a linearised stability problem. Let

$$
\psi_{t}=L_{R} \psi+N(\psi), \psi(x, y, t): \mathbf{R}^{n} \times \Omega \times \mathbf{R}^{+} \rightarrow \mathbf{R}^{N},
$$

describe the 'full' problem, where $L_{R}$ (respectively $N$ ) is a linear (nonlinear) operator, $R$ is a control, or bifurcation, parameter and $\Omega$ is a bounded domain $\subset \mathbf{R}^{m}$. For simplicity we assume that $n=1$. The linearised stability of the basic solution $\psi_{0}(y)$ of (1.2) is determined by setting

$$
\psi=\psi_{0}+f(y) e^{i k x+\mu t}
$$

and solving, for any pair $(k, R)$, an eigenvalue problem for $f(y)$ with eigenvalues $\mu=\mu(k, R)$. Define $\mu_{0}(k, R)$ as the critical eigenvalue (that is, the eigenvalue with the largest real part) for a given pair $(k, R)$ and $R_{c}$ as the critical bifurcation value of $R: \operatorname{Re} \mu_{0}(k, R)<0$ for all $k \in \mathbf{R}$ and $R<R_{c}$, i.e. the basic solution $\psi_{0}$ of (1.2) is linearly stable for $R<R_{c}$. The curve in the $(R, k)$-plane where $\operatorname{Re} \mu_{0}(k, R)=0$ is the so-called neutral curve. The critical values $k_{c}$ of $k$ and $w_{c}$ of $\mu_{0}(k, R)$ are defined by

$$
\mu_{0}\left(k_{c}, R_{c}\right)=i w_{c}
$$

$f_{c}(y)$ is the critical eigenfunction at $R=R_{c}, k=k_{c}$. If $R$ is 'slightly' above $R_{c}$, i.e. $R=R_{c}+r \varepsilon^{2}$ with $0<\varepsilon \ll 1, r>0$, one makes the following basic 'Ansatz' on which the derivation of the GL-equation is based

$$
\psi(x, y, t)=\psi_{0}(y)+\varepsilon A(\xi, \tau) f_{c}(y) e^{i\left(k_{c} x+w_{c} t\right)}+\text { c.c. }
$$

where $\xi$ and $\tau$ are rescaled time and space coordinates. Equation (1.3) gives only the leading order term of a double expansion: as a Taylor series in $\varepsilon$

and as a Fourier series in $e^{i\left(k_{c} x+w_{c} t\right)}$. The GL-equation (1.1) describes the modulation of the unknown amplitude $A(\xi, \tau)$ (see section 3.1 for more details). In the last 10 years much progress has been made in the mathematical 
justification of this approach. Eckhaus [15] showed that the above 'Ansatz' can be made rigorous for a large class of model systems. The validity of the GL equation for various types of model equations has been shown by many authors (see $[5,17,3,26]$ ).

The fact that $\psi(x, y, t)-\psi_{0}(y)$ can be expanded in a Fourier series in $e^{i\left(k_{c} x+w_{c} t\right)}$ is a fundamental 'non-degeneracy condition' on which both the asymptotic process leading to the GL-equation and the proof of its validity is based. It is clear that the Fourier expansion must break down when $k_{c}=w_{c}=0$. A priori this might seem like a phenomenon of high codimension, but this is not necessarily the case: $w_{c}=0$ is a 'generic' property of basic reversible systems (1.2), i.e. systems of PDE's that are symmetric with respect to the transformation $x \rightarrow-x$. Reversible systems are very natural, a classical example is convection (see for instance [23], one of the first papers in which a GL-equation has been derived). If $w_{c}=0$, then one only needs one extra bifurcation parameter, $s$, to make the GL Ansatz (1.3) degenerate, i.e. there must be a special value of $s$ such that $k_{c}(s)=0$.

In this paper we make an asymptotic analysis of the behaviour of small solutions near such a co-dimension 2 point: a point in the two-dimensional parameter plane at which the classical GL Ansatz (1.3) breaks down. In remark 1.1 we discuss the relation of the co-dimension 2 point studied in this paper to other, similar, co-dimension 2 degenerations that occur in the derivation of the GL-equation. Such co-dimension 2 degenerations occur in convection problems, nematics, lasers, etc. $([30,2,21,22])$.

Instead of performing this analysis on a very general (reversible) model problem as (1.2), we focus on a more simple, but highly relevant 'basic' problem, a general reversible two-dimensional system of reaction-diffusion equations:

$$
\left\{\begin{array}{l}
u_{t}=r u+c_{1} v+u_{x x}+N_{1}(u, v) \\
v_{t}=s v+c_{2} u+d v_{x x}+N_{2}(u, v),
\end{array}\right.
$$

where $x \in \mathbf{R} ; N_{1}$ and $N_{2}$ are analytic non-linear expressions (thus we assume that $N_{i}(u, v), i=1,2$, do not have any linear terms). The system will be studied by varying the bifurcation parameters $r$ and $s$; the other constants, $c_{1}, c_{2}$ and $d$, will be kept fixed. If we compare (1.4) to (1.2) we note that $\psi(x, y, t)=(u(x, t), v(x, t))^{\mathrm{T}}$, thus, there is no $y$ variable (i.e. $\left.\Omega=\emptyset\right)$ : this simplifies the linear stability analysis considerably. Moreover, the model problem is chosen in such a way that $\psi_{0}(y) \equiv(0,0)^{\mathrm{T}}$.

It should be remarked that studying the weakly non-linear stability of a 'trivial pattern' $(\hat{u}(\hat{x}, t), \hat{v}(\hat{x}, t)) \equiv\left(\hat{U}_{0}, \hat{V}_{0}\right)$, where $(\hat{u}(\hat{x}, t), \hat{v}(\hat{x}, t))$ is a solu- 
tion of the, a priori, more general system

$$
\left\{\begin{array}{l}
\hat{u}_{t}=d_{1} \hat{u}_{\hat{x} \hat{x}}+f_{1}(\hat{u}, \hat{v}) \\
\hat{v}_{t}=d_{2} \hat{v}_{\hat{x} \hat{x}}+f_{2}(\hat{u}, \hat{v})
\end{array}\right.
$$

is completely equivalent to studying the weakly non-linear stability of the trivial, basic pattern $(0,0)$ of (1.4). This can be seen by setting $(\hat{u}(\hat{x}, t), \hat{v}(\hat{x}, t))=\left(\hat{U}_{0}+u(\hat{x}, t), \hat{V}_{0}+v(\hat{x}, t)\right)$ and substituting this into (1.5) - $\hat{x}$ can be rescaled to $x$ in such a way that $d_{1}=1$. Thus, model problem (1.4) can also be used to describe the GL bifurcation and its degeneration in well-known models as the Brusselator (see [20] for the derivation of the GL-equation in the Brusselator), the Gray-Scott model [10], systems of predator-prey type [6] etc. Moreover, our basic model is as general as the system studied by Turing [29], although it should be noted that in that paper the equations are studied on a bounded $x$-domain, while it is crucial for the forthcoming GL-analysis that $x \in \mathbf{R}$.

Another additional advantage of studying (1.4) instead of (1.2) is that it is now quite easy to check the asymptotic computations by a numerical simulation of the full basic system (1.4)-see section 5 .

If $c_{1} c_{2}<1$ there is a curve $\Gamma_{G L}$ in the $(r, s)=R$-parameter plane at which the GL bifurcation occurs, i.e. the basic pattern $(0,0)$ becomes unstable and the evolution of small solutions can be described by 'Ansatz' (1.3) and the GL-equation (1.1) where now $c, h \in \mathbf{R}$, due to the reversibility symmetry, and $\Delta=\frac{\partial^{2}}{\partial \xi^{2}}$. From now on we rescale to $c_{1}=1, c_{2}=-1$ to assure that $c_{1} c_{2}<0$. However, $k_{c}=k_{c}(s)$ and there is a point $\left(r_{e F K}, s_{e F K}\right)$ where $k_{c}=0$ (and $w_{c}=0$; in section 2 we will also encounter a point $\left(r_{c G L}, s_{c G L}\right)$ where $k_{c}=0$ but $w_{c} \neq 0$, see remark 1.2). On one side of the point $\left(r_{e F K}, s_{e F K}\right)$ on $\Gamma_{G L}, k_{c} \neq 0$ exists, on the other side $k_{c} \equiv 0$.

In an $O(\delta), 0<\delta \ll 1$, neighbourhood of the point $\left(r_{e F K}, s_{e F K}\right)$ one has to replace the GL Ansatz (1.3) by the following Ansatz:

$$
\left(\begin{array}{l}
u \\
v
\end{array}\right)=\delta^{2}\left(\begin{array}{c}
\sqrt{d} \\
1
\end{array}\right) B\left(\xi_{B}, \tau_{B}\right)+O\left(\delta^{3}\right),
$$

this is only a Taylor expansion in $\delta$, but no longer a Fourier expansion. Note also that the unknown 'amplitude' $B\left(\xi_{B}, \tau_{B}\right)$ is real valued, while $A(\xi, \tau)$ was complex. Moreover, the scalings of $\xi_{B}$ and $\tau_{B}$ differ from those of $\xi$ and $\tau$ (see sections 3.1 and 3.2). Based on this Ansatz one can derive a so-called extended Fisher-Kolmogorov (eFK) equation for $B$, that reads in rescaled form

$$
B_{\tau_{B}}=B+D B_{\xi_{B} \xi_{B}}-B_{\xi_{B} \xi_{B} \xi_{B} \xi_{B}}-B^{2},
$$


where $D$ still depends on $r$ and $s: D<0$ as $k_{c} \neq 0$ exists, $D=0$ at $\left(r_{e F K}, s_{e F K}\right)$ and $D>0$ as $k_{c}=0$. Note that the name-giving of equation (1.7) is in the literature somewhat confusing. Here we follow [24, 8, 18, 7]. However, the name eFK-equation is usually given to equations of the type (1.7) with $D>0$ and a cubic instead of a quadratic non-linearity. In the case $D<0$ the name Swift-Hohenberg (SH) equation would be more appropriate ([28]), however, the SH-equation also has a cubic non-linearity in general. Since the transition at $\left(r_{e F K}, s_{e F K}\right)$ from $D>0$ to $D<0$ is most naturally described by an equation in the form of a (quadratic) eFK-equation we prefer in this paper the name eFK2 for equation (1.7). We will also encounter the more standard cubic eFK or SH-equation as a degenerate case of the eFK2-equation (1.7) and call it the eFK3-equation.

The derivation of the eFK2-equation is quite straightforward. However, if one compares the (complex) GL-equation (1.1) to the (real, fourth order) eFK2-equation, then it is a priori hard to grasp how the transition from the GL-equation to the eFK2-equation should take place. Understanding this transition is the main subject of this paper.

Although we do not approach this problem by transforming it completely into Fourier space (as for instance in [17]), we will use the interpretation of the GL Ansatz (1.3) and the eFK Ansatz (1.6) in Fourier space to study the transition. The GL Ansatz is represented in Fourier space by sharp disjunct 'peaks' around the values $n k_{c}, n=0, \pm 1, \pm 2, \ldots$ (see figure 4 , section 4.2 ), while the eFK Ansatz is described by a wide, solitary, 'peak'. It is shown in this paper that the GL Ansatz breaks down as $k_{c} \downarrow 0$ and the formerly disjunct peaks start to overlap. The wide eFK peak then appears as the envelope of the overlapping GL peaks (figure 5, section 4.2).

However, this is only a part of the full picture. We will show that there is a region on $\Gamma_{G L}$ near the co-dimension 2 point $\left(r_{e F K}, s_{e F K}\right)$ at which both the GL-equation and the eFK2-equation are valid. In this region these equations both describe the evolution of small solutions, but the scales of the magnitude, and the temporal and spatial evolution differ significantly, i.e. the GL and the eFK2-equation describe different patterns. Moreover, the GL-equation should be replaced by a singularly perturbed GL-equation:

$$
A_{\tau_{A}}=r A+c_{e F K} A_{\xi_{A} \xi_{A}}+\frac{h_{e F K}}{1-d} A|A|^{2}-\frac{4 i d \gamma}{1-d} A_{\xi_{A} \xi_{A} \xi_{A}}-\frac{d \sqrt{d} \gamma^{2}}{1-d} A_{\xi_{A} \xi_{A} \xi_{A} \xi_{A}}
$$

(at leading order), where $0<\gamma=\frac{\varepsilon}{\delta} \ll 1$ and $c_{e F K}, h_{e F K}$ are (rescaled) limits of the values of $c, h$ of (1.1) at $\left(r_{e F K}, s_{e F K}\right)$. Here something very interesting occurs. It follows from the asymptotic analysis that

$$
h_{e F K} \geq 0,
$$


independent of the non-linear terms $N_{1}(u, v)$ and $N_{2}(u, v)$ in (1.4). This is interesting because the (non-singularly perturbed) GL-equation (1.1) (in one dimension) does not have bounded periodic solutions if $h>0$. Moreover, solutions have a tendency to blow up (in finite time) in this case. It follows immediately in the numerical simulations of the full system (1.4) that small $O(\varepsilon)$ solutions (see (1.3)) grow towards an $O(1)$ magnitude if $h>0$, which means that the weakly non-linear GL approach is only valid on very small time intervals and cannot be used to study the asymptotic behaviour of patterns. Thus, as in almost all applications and studies of the GL-equation (see [25] for a review), one prefers a GL-equation with a negative Landau coefficient $h$ when describing the evolution of small patterns near $\Gamma_{G L}$. If $(r, s)$ is near $\Gamma_{G L}$ but not near $\left(r_{e F K}, s_{e F K}\right)$, this can be achieved for a 'generic choice' of $N_{1}(u, v)$ and $N_{2}(u, v)$ (see section 4.1). One then observes stationary, stable periodic patterns in the numerical simulations of the full system (1.4), exactly as predicted by the GL-equation. However, since we found that $h_{e F K} \geq 0$ we know that there must be a point $\left(r_{n l}, s_{n l}\right)$ on $\Gamma_{G L}$ where $h=0$. This can be called a non-linear bifurcation.

This bifurcation was first studied in $[16,9]$ and later more rigorously in [27]. To give a correct description of the behaviour of solutions in this case one needs to consider extra non-linear terms of higher order (such as $A|A|^{4}$ and $A_{\xi}|A|^{2}$ - see section 4.1) to obtain a degenerate GL-equation of a form as given in (4.5). If the $\left(r_{n l}, s_{n l}\right)$ is not close to $\left(r_{e F K}, s_{e F K}\right)$ then there is a part of $\Gamma_{G L}$ where the GL approach is valid and the GL equation has a positive Landau coefficient: here there are no bounded small solutions. Thus, since we are interested in bounded small solutions, we have to choose $\left(r_{n l}, s_{n l}\right)$ so close to $\left(r_{e F K}, s_{e F K}\right)$ that the eFK approach is already valid between $\left(r_{n l}, s_{n l}\right)$ and $\left(r_{e F K}, s_{e F K}\right)$.

We did not study this situation in its full asymptotic details (see figures 8, 9 and 10 for the numerical simulations and section 5.4 for a discussion), but, we did consider the special case $h_{e F K}=0$. This can quite easily be achieved by considering non-linearities $N_{1}(u, v)$ and $N_{2}(u, v)$ in (1.4) of cubic or higher order. Is this case we find that the behaviour of small solutions near $\left(r_{e F K}, s_{e F K}\right)$ is governed by a cubic (!) eFK-equation, the eFK3 (or $\mathrm{SH})$ equation:

$$
B_{\tau_{B}}=B+D B_{\xi_{B} \xi_{B}}-B_{\xi_{B} \xi_{B} \xi_{B} \xi_{B}}+l B^{3},
$$

where $D$ is as in (1.7). The sign of $l$ can now be both positive or negative, as function of the structure of $N_{1}(u, v)$ and $N_{2}(u, v)$ (see section 3.4). Thus, although the assumption that both $N_{1}(u, v)$ and $N_{2}(u, v)$ are cubic makes the problem of a higher co-dimension, it is a natural assumption: this way there are no 'problems' with solutions that cannot be described (for all time) by the weakly non-linear theory. Moreover, it enables us to derive equations 
that appear throughout the literature $([24,8,28,18,7])$.

As already mentioned above, we supplement the asymptotic analysis of the transition from the GL-equation to the eFK-equation by a numerical study of the behaviour of small solutions of the full system (1.4) near $\Gamma_{G L}$, and of course especially near $\left(r_{e F K}, s_{e F K}\right)$. Since we choose $N_{1}(u, v)$ and $N_{2}(u, v)$ such that $h<0$ we can first check the validity of the GL approach: we find stable periodic solutions as predicted by the Eckhaus stability criterion ([12]). By bringing $(r, s)$ closer and closer to $\left(r_{e F K}, s_{e F K}\right)$, we observe stable, stationary patterns of a much richer structure than the sine/cosine like periodic patterns described by the (real) GL-equation (see figures 8, 9 and 10).

These solutions have a clear 'multi-bump' structure. In figure 1 we show two examples of (small) numerically stable 'multi-bump' solutions of the full reaction-diffusion system (1.4). Parameters $r$ and $s$ are close to $\left(r_{e F K}, s_{e F K}\right)$ and $N_{1}(u, v)$ and $N_{2}(u, v)$ are such that the solutions are (asymptotically) described by the eFK3-equation. Solutions like these have been and still are the subject of much ongoing research (see for instance [4] and the references given there). In these papers the existence of solutions similar to those observed as stable patterns of the reaction-diffusion system (1.4) near the co-dimension 2 point, are shown to exist for the stationary problems associated to either the eFK2 ([4]) or the eFK3-equation ([24, 18]). However, it should be noted, that there are no proofs (yet) of the stability of these stationary 'multi-bump' solutions as solutions to the full eFK PDE. Nevertheless, figure 12a shows a typical example of a stable multi-bump pattern that appears by integrating the eFK3-equation: note that these patterns are quite similar to those shown in figure 1 . We refer to section 5.4 for a more detailed discussion.

Thus, the asymptotic and numeric calculations both strongly suggest that the 'multi-bump' solutions to the eFK2,3-equations can be asymptotically stable and that the 'attractors' in these equations also are of significant importance to the behaviour of small solutions near the co-dimension 2 point at which the $k_{c}$ of the GL Ansatz (1.3) becomes 0 (or small). However, both steps are not proven. The results of this paper are based on a detailed analysis of the reaction-diffusion system (1.4). The essence of the method in this paper does not depend on the exact structure of the underlying model problem. Therefore, the analysis in this paper can also be applied to much more general systems with a similar co-dimension 2 degeneration. As a consequence, it can be expected that the asymptotic stable 'multi-bump' patterns encountered in this paper, will also occur in these more general model problems.

Remark 1.1 There exist some related co-dimension 2 bifurcations studied 
in the literature. Two of those are quite similar to the bifurcation studied in this paper. In [21] and [22] a complete weakly non-linear description is given of a co-dimension 2 bifurcation that appears in laser dynamics. As in this paper, $k_{c}=w_{c}=0$ at the co-dimension 2 point described by $\Omega=0$ : the 'detuning' $\Omega$ plays a role similar to $s$ in this paper. However, unlike the eFK-bifurcation studied here, $w_{c}(\Omega) \neq 0$ for $\Omega \neq 0$. As a result, the dynamics near threshold are described by different kinds of cubic and complex Swift-Hohenberg equations. Another co-dimension 2 point is the so-called Lifshitz point that for instance appears in (planar) nematics (see for [30, 2]). This is a purely two dimensional (i.e. in $(x, y)$-space) phenomenon: the critical point of the neutral surface at $k=k_{c}, l=0$ bifurcates into two critical points at $k=k_{c}^{\prime}, l= \pm l_{c} \neq 0([2])$. In this case the modulation equation for the amplitude $A(\xi, \eta, \tau)$ is once again cubic and complex, it reduces to the eFK3-equation if one considers a real amplitude $A$, independent of $\xi$ ([30]). There are many more possible co-dimension 2 degenerations, we do not intend to try to give a complete list here; see for instance [31, 11], and the references given there, for co-dimension 2 bifurcations in convection problems.

Remark 1.2 As noted above, we will also encounter the co-dimension 2 point $\left(r_{c G L}, s_{c G L}\right)$ on $\Gamma_{G L}$ where $k_{c}=0$, but $w_{c} \neq 0$. In section 3.3 we briefly sketch how this case can be described by a coupled system of GL equations (see [11] and the references given there). Numerically we observe that the stable periodic patterns described by (1.1) obtain a periodically modulated amplitude near the point $\left(r_{c G L}, s_{c G L}\right)$, see figure 7 a.

Remark 1.3 The structure of the paper is as follows: in section 2 we study the linear stability of the trivial solution $(0,0)$ to $(1.4)$, with $c_{1}=1$, $c_{2}=-1$. The derivations of the GL, the eFK and some other relevant modulation equations are given in section 3 (and appendix $\mathrm{A}$ ). The main subject of the paper, the transition from the GL to eFK2-equation is studied in section 4 . In section 5 we present and interpret the numerical simulations.

\section{The linear stability analysis}

We start by performing a linear stability analysis for the solution $(u, v)=$ $(0,0)$ of the reaction-diffusion system (1.4) where $c_{1}=1, c_{2}=-1$ :

$$
\left\{\begin{array}{l}
u_{t}=r u+v+u_{x x}+N_{1}(u, v) \\
v_{t}=s v-u+d v_{x x}+N_{2}(u, v) .
\end{array}\right.
$$

We study the stability of the solution by substituting

$$
\left(\begin{array}{l}
u \\
v
\end{array}\right)=e^{i k x+\omega t}\left(\begin{array}{c}
\tilde{u} \\
\tilde{v}
\end{array}\right)
$$


into the linear part of the system. This gives the following eigenvalue problem

$$
\omega\left(\begin{array}{c}
\tilde{u} \\
\tilde{v}
\end{array}\right)=\left(\begin{array}{cc}
r-k^{2} & 1 \\
-1 & s-d k^{2}
\end{array}\right)\left(\begin{array}{c}
\tilde{u} \\
\tilde{v}
\end{array}\right) .
$$

Studying the linearised stability of $(0,0)$ reduces to calculating the eigenvalues $\omega$ of this $2 \times 2$ eigenvalue problem. The solution $(0,0)$ is stable as long as the real parts of both eigenvalues are negative for all $k \in \mathbf{R}$ and becomes unstable when the real part of one of the eigenvalues becomes positive (for some $k \in \mathbf{R}$ ). The characteristic polynomial reads

$$
\omega^{2}-\left(s+r-(1+d) k^{2}\right) \omega+r s+1-(r d+s) k^{2}+d k^{4}=0 .
$$

This leads to two eigenvalues $\omega_{1}$ and $\omega_{2}$, where we assume that $\operatorname{Re}\left(\omega_{1}\right) \geq$ $\operatorname{Re}\left(\omega_{2}\right)$. Moreover, we define for fixed $s$ and $d$ the neutral curve $\left\{\operatorname{Re} \omega_{1}(k, r)=\right.$ $0\}$. When we plot this curve in the $(k, r)$-plane, we know that the solution $(0,0)$ is stable against perturbations of the type $(2.2)$ for $(k, r)$ outside the neutral curve; perturbations grow exponentially when $(k, r)$ is inside the neutral curve.

In order to study this neutral curve, we need to know more about the real part of the two eigenvalues. We start with looking for the critical points $\left(k_{c}, r_{c}\right)$ of the neutral curve. Here the $r_{c}$ still depends on $s$. First we assume that $\omega$ is real near $\left(k_{c}, r_{c}\right)$. By definition we know that $\omega\left(k_{c}, r_{c}\right)=$ $\frac{\partial \omega}{\partial k}\left(k_{c}, r_{c}\right)=0$. Setting $\omega\left(k_{c}, r_{c}\right)=0$ in $(2.4)$ leads to

$$
d k_{c}^{4}-\left(r_{c} d+s\right) k_{c}^{2}+r_{c} s+1=0 .
$$

Applying $\frac{\partial}{\partial k}$ to $(2.4)$ and substituting $\omega\left(k_{c}, r_{c}\right)=\frac{\partial \omega}{\partial k}\left(k_{c}, r_{c}\right)=0$ gives

$$
k_{c}\left(4 d k_{c}^{2}-2\left(r_{c} d+s\right)\right)=0 .
$$

Therefore

$$
k_{c}=0 \vee k_{c}^{2}=\frac{r_{c} d+s}{2 d},
$$

where $r_{c}$ has yet to be determined. The second pair of critical values only exists when $\frac{r_{c} d+s}{2 d} \geq 0$. It follows from (2.4) that $k_{c}=0$ is the only critical value if $\omega\left(k_{c}, r_{c}\right) \notin \mathbf{R}$.

Thus, we can distinguish between different types of instabilities. As long as $\frac{r_{c} d+s}{2 d}>0$ the eigenvalue-curve, where $\operatorname{Re}\left(\omega_{1}\right)$ is given as a function of $k$ for fixed $(r, s)$, has two maxima in $k_{c}= \pm \sqrt{\frac{r_{c} d+s}{2 d}}$ and one minimum in $k=0$ (see figure 2c). This can be seen as follows: for $|k| \gg 1,(2.4)$ implies, by taking only all the terms of the highest order, that $\omega^{2}+(1+d) k^{2} \omega+d k^{4} \sim 0$. Thus $\omega \sim-k^{2}$ or $\omega \sim-d k^{2}$, therefore, for $|k| \gg 1$, the real parts of the eigenvalues are negative. The first solution to become unstable, for this 
eigenvalue-curve, is the wave $e^{i k_{c} x}$, where $k_{c}=\sqrt{\frac{r_{c} d+s}{2 d}}$.

We now note that a co-dimension 2 bifurcation occurs, where $s$ is the second bifurcation parameter, for $\frac{r_{c} d+s}{2 d}=0$ : the curve has only one maximum in $k=0$ for $\frac{r_{c} d+s}{2 d}<0$. Here, the first solution to become unstable is the 'wave' with wavenumber $k=0$ : $e^{i 0 x}$. Thus, for fixed $r=r_{c}$ and varying the second bifurcation parameter $s$ we see that this bifurcation occurs at $s=s_{e F K}$ with $r_{c} d+s_{e F K}=0$. For $s>s_{e F K}$, the curve has two maxima and for $s<s_{e F K}$ the curve has one maximum (see figures $2 \mathrm{a}, \mathrm{b}$ and $\mathrm{c}$ ). Since we assume that the first bifurcation occurs at $\left(k_{c}, r_{c}\right)$ we have to set $\operatorname{Re} \omega_{1}\left(k, r_{c}\right) \leq 0$ and $\operatorname{Re} \omega_{2}\left(k, r_{c}\right)<0$ for every $k \in \mathbf{R}$, this implies that $\omega_{1}+\omega_{2} \leq 0$. Combining this with the fact that (2.4) can be factorised as $\left(\omega-\omega_{1}\right)\left(\omega-\omega_{2}\right)=\omega^{2}-\left(\omega_{1}+\omega_{2}\right) \omega+\omega_{1} \omega_{2}=0$, leads to $s+r_{c}-(1+d) k^{2} \leq 0$ for every $k \in \mathbf{R}$. From this it follows that

$$
s+r_{c} \leq 0 .
$$

We now have the two conditions under which the first bifurcation occurs: $r+s<0$ and $r d+s>0$. It depends on the magnitude of $d$ in which region of the $(r, s)$-plane these conditions hold. For $d<1$, they hold in the second quadrant of the $(r, s)$-plane $(r<0$ and $s>0)$ and for $d>1$ they hold in the fourth quadrant. From now on we choose $d<1$, this does not influence the results. This choice fixes the signs of $r$ and $s$, namely $r<0$ and $s>0$. In figure 3 , we sketch the second quadrant of the $(r, s)$-plane with the bifurcation curves obtained for $d<1$. There is a bifurcation curve $\Gamma_{G L}$ which consists of three parts: $\Gamma_{1}, \Gamma_{2}, \Gamma_{3}$. The different eigenvalue-curves occur on these three parts. On $\Gamma_{1}$ the eigenvalue-curve has one maximum and on $\Gamma_{2}$ it has two maxima. We will now determine the form of the eigenvalue-curve on $\Gamma_{3}$.

If $s+r_{c}=0$, then $\operatorname{Re} \omega_{1}\left(0, r_{c}\right)=\operatorname{Re} \omega_{2}\left(0, r_{c}\right)=0$ and the eigenvaluecurve has three maxima, in $k=0$ and in $k= \pm k_{c}$, where $\operatorname{Re} \omega_{1}=0$ in all three maxima. Therefore another co-dimension 2-bifurcation occurs at $s=s_{c G L}$ with $s_{c G L}+r_{c}=0$. Here the eigenvalue-curve transforms from a curve with two maxima, through a curve with three maxima, to a curve with one maximum in $k=0$ (see figures $2 \mathrm{~d}$, e and $\mathrm{f}$ ). To be able to sketch the eigenvalue-curves we also need to know where the eigenvalues are real and where they become complex. Note that the eigenvalues are always real in a neighbourhood of $k=k_{c} \neq 0$ and for $|k| \gg 1$. Since the eigenvalue-curves are symmetric in $k$, the first $k$-value for which they can become complex is for $k=0$ (see (2.4)). Setting $k=0$ in (2.4) and solving $\omega$ from that equation gives

$$
\omega_{1,2}=\frac{1}{2}\left(s+r \pm \sqrt{(s-r)^{2}-4}\right) .
$$

Thus the eigenvalues become complex in $k=0$ when $(s-r)^{2}-4=0$. This implies that the eigenvalues are complex in some interval of $k$-values for 
$s-2<r<s+2$. We define $\tilde{s}$ as the value of $s$ at which the neutral curve at $r=r_{c}$ becomes complex at $k=0$. Since $r<0$ and $s>0$, the eigenvalues become complex for $r=s-2$ at $s=\tilde{s}=\frac{2 \sqrt{d}}{1+\sqrt{d}}$. For $s<\tilde{s}$, all eigenvalues are real. Complex eigenvalues exist for $s>\tilde{s}$.

Of course we still have to determine $r_{c}$. We can calculate $r_{c}$ from (2.5) for the different choices of $s$. For $s<s_{e F K}$ and on the bifurcation curve $\Gamma_{1}$, we have that $k_{c}=0$ and so we get that

$$
r_{c}=-\frac{1}{s}
$$

By substitution of $k_{c}= \pm \sqrt{\frac{r_{c} d+s}{2 d}}$ in (2.5), we can determine the critical $r$ value $r_{c}$ which belongs to the $s$-values on the bifurcation curve $\Gamma_{2}\left(s_{e F K}<\right.$ $\left.s<s_{c G L}\right)$. We obtain that

$$
r_{c}=\frac{s}{d}-2 \sqrt{\frac{1}{d}},
$$

where we chose the minus-sign because $r<0$. Note that from this calculation it follows that $c_{1} c_{2}$ has to be negative in system (1.4). This can be seen from the equation (2.5) with general $c_{1}$ and $c_{2}$, instead of $c_{1}=1$ and $c_{2}=-1$

$$
d k_{c}^{4}-\left(r_{c} d+s\right) k_{c}^{2}+r_{c} s-c_{1} c_{2}=0 .
$$

Substituting $k_{c}^{2}=\frac{r_{c} d+s}{2 d}$ leads to

$$
\left(r_{c} d-s\right)^{2}=-4 d c_{1} c_{2},
$$

from which can be seen that $c_{1} c_{2}<0$ has to hold. Thus, if $c_{1} c_{2}>0$ the above described bifurcations do NOT occur. On the curve $\Gamma_{3}$ we know that $\operatorname{Re} \omega_{1}\left(0, r_{c}\right)=\operatorname{Re} \omega_{2}\left(0, r_{c}\right)=0$, thus we deduce from (2.6) that

$$
r_{c}=-s
$$

Now, we can determine the above defined $s_{e F K}$ and $s_{c G L}$. It follows from $r_{c} d+s_{e F K}=0$ that

$$
s_{e F K}=\sqrt{d} .
$$

From $r_{c}+s_{c G L}=0$ we obtain that

$$
s_{c G L}=\frac{2 \sqrt{d}}{1+d} .
$$




\section{The modulation equations}

So far we showed that when varying $(r, s)$ along the bifurcation curve $\Gamma_{G L}$, the eigenvalue curves (the real part of the eigenvalues) change smoothly from a curve with one maximum at $k=0$ (on $\Gamma_{1}$, for $s<s_{e F K}$ ) to a curve with two maxima at $k= \pm k_{c}$ and a minimum at $k=0$ (on $\Gamma_{2}$, for $s_{e F K}<s<s_{c G L}$ ) to again a curve with one maximum at $k=0$ (on $\Gamma_{3}$ ), see figure 2. For these different cases, modulation equations can be derived by weakly non-linear stability analysis. Because the eigenvalue-curve goes smoothly from one type to another type, the derived equations are limits of each other. First we derive the GL-equation on $\Gamma_{2}$ for $s_{e F K}<s<s_{c G L}$. Then there are two transitions, they occur at $s=s_{e F K}$ and at $s=s_{c G L}$. In this paper we will not study the transition at $s=s_{c G L}$. Decreasing $s$ so that it is close to $s_{e F K}$ leads to the eFK-equation as modulation equation. Decreasing $s$ further to $s \ll s_{e F K}$ on $\Gamma_{1}$ gives the Fisher-Kolmogorov equation. For $s$ close to $s_{c G L}$ a coupled system of two GL-equations can be derived (this explains the index 'cGL': 'coupled Ginzburg-Landau'); and for $s \ll s_{C G L}$ on $\Gamma_{3}$ we again obtain a GL-equation. However, the coefficients here are complex. In this section we will derive all relevant modulation equations.

For $r<r_{c}$ the real part of the eigenvalue $\omega_{1}$ becomes positive for an interval of $k$-values, which means that for these $k$-values the solution is unstable. The modulation equations to be derived in this section describe the behaviour of 'small solutions' for $r<r_{c}$ and $r-r_{c} \ll 1$. We consider as non-linear terms in (2.1)

$$
\begin{aligned}
& N_{1}(u, v)=\alpha_{1} u^{2}+\alpha_{2} u v+\alpha_{3} v^{2}-a u^{3} \\
& N_{2}(u, v)=\beta_{1} u^{2}+\beta_{2} u v+\beta_{3} v^{2}-b v^{3} .
\end{aligned}
$$

This choice is not at all essential, we can take more (general) terms into account but then only the calculations become more extensive: new terms do not influence the derivation process, they only alter the coefficients of the

modulation equation. Studying the stability of $(0,0)$ is done by studying the eigenvalue problem (2.3). We define

$$
\mathcal{M}_{c}=\left(\begin{array}{cc}
r_{c}-k_{c}^{2} & 1 \\
-1 & s-d k_{c}^{2}
\end{array}\right) .
$$

Now we can derive the modulation equation(s) for different choices of $s$.

Remark 3.1 General analytic non-linear terms $N_{i}(u, v)$ can be expanded into a power series in $u$ and $v$. Expressions (3.1) and (3.2) can then be seen as the first four terms in these expansions. It is easy to check that only the quadratic and the cubic terms in $N_{i}(u, v)$ are relevant in the derivation of the modulation equations. 


\subsection{The GL-equation}

We start by setting $s_{e F K}<s<s_{c G L}$ (and $s$ not close to $s_{e F K}$ ) along $\Gamma_{2}$, then a standard, real GL-equation can be derived. Although the derivation is quite standard and straightforward we present it here in some detail so that it can serve as background for the subsequent sections. Here $r_{c}=\frac{s}{d}-\frac{2}{\sqrt{d}}$ and $k_{c}=\sqrt{\frac{r_{c} d+s}{2 d}}=\sqrt{\frac{s-\sqrt{d}}{d}}$, therefore

$$
\mathcal{M}_{c}=\left(\begin{array}{cc}
-\frac{1}{\sqrt{d}} & 1 \\
-1 & \sqrt{d}
\end{array}\right) \text {. }
$$

The eigenvalues of this matrix $\mathcal{M}_{c}$ are $\lambda_{1}=0$ and $\lambda_{2}=-\frac{1-d}{\sqrt{d}}<0$ and the corresponding eigenvectors are $w_{1}=\left(\begin{array}{c}\sqrt{d} \\ 1\end{array}\right)$ resp. $w_{2}=\left(\begin{array}{c}\frac{1}{\sqrt{d}} \\ 1\end{array}\right)$. We will see that a modulation equation appears as a consequence of a certain solvability condition. Here, the solvability condition is given by the equation $\mathcal{M}_{c} x=b$. This equation can only be solved when $b \in S p\left\{w_{2}\right\}$. Thus, if we write $b=\left(\begin{array}{l}b_{1} \\ b_{2}\end{array}\right)$ we find the solvability condition

$$
b_{1}-\frac{1}{\sqrt{d}} b_{2}=0 .
$$

In the following sections, we will find that $\mathcal{M}_{c}$ is, in highest order, always as given above and thus the solvability condition is also the same in all derivations.

As is standard in the derivation of the GL-equation, we take $r$ close to $r_{c}: r=r_{c}-v_{1} \varepsilon^{2}$ where $0<\varepsilon \ll 1$ and $v_{1}>0$. In the non-linear stability analysis we model the perturbation of the solution $(0,0)$ as a slow modulation of the wave with wavenumber $k=k_{c}=\sqrt{\frac{s-\sqrt{d}}{d}}$, the "most unstable wave':

$$
\left(\begin{array}{l}
u \\
v
\end{array}\right)=\varepsilon\left(\begin{array}{c}
\sqrt{d} \\
1
\end{array}\right) A(\xi, \tau) e^{i k_{c} x}+\text { c.c. }+ \text { hot },
$$

here $\left(\begin{array}{c}\sqrt{d} \\ 1\end{array}\right)$ is the 'most critical direction', it is the eigenvector which belongs to the eigenvalue $\lambda_{1}=0$. The slow space and time variables $\xi$ and $\tau$ are scaled in a standard way, $\xi=\varepsilon x$ and $\tau=\varepsilon^{2} t$ (see [15] for a rigorous foundation of these scalings). We will explain the scaling of $\xi$ a bit further. Classically, this scaling is related to the width of the interval of $k$-values for which the solution $(0,0)$ is unstable at $r=r_{c}-v_{1} \varepsilon^{2}$. We have to determine for which $k$ the eigenvalue-curve of the largest eigenvalue 
intersects the $k$-axis. Substituting $\omega=0$ and $r=r_{c}-v_{1} \varepsilon^{2}$ into (2.4) leads to

$$
d k^{4}-\left(2 s-2 \sqrt{d}-v_{1} d \varepsilon^{2}\right) k^{2}+\left(\frac{s}{\sqrt{d}}-1\right)^{2}-v_{1} s \varepsilon^{2}=0 .
$$

This equation has four solutions: $k= \pm k_{c} \pm \frac{\varepsilon}{2} \sqrt{\frac{\sqrt{d} v_{1}}{s-\sqrt{d}}}$. Therefore the width of the interval of the unstable $k$-values is of $O(\varepsilon)$ and we scale $\xi=\varepsilon x$. Note that for $s \rightarrow s_{e F K}=\sqrt{d}$, the term $s-\sqrt{d}$ becomes small, which changes the width of the interval of unstable $k$-values and thus the scaling of $\xi$ changes, see section 3.2. The relevant scalings of $\xi, \tau$ and the magnitude of the perturbation of solution $(0,0)$ can also be deduced by using the significant degeneration method (see [13]).

The non-linear terms in (2.1) will generate harmonics of the simple linear wave $e^{i k_{c} x}$. Thus the higher order terms in the expansion of the perturbation are constructed from a product of this most unstable wave with itself,

$$
\left(\begin{array}{c}
u \\
v
\end{array}\right)=e^{i k_{c} x}\left(\varepsilon\left(\begin{array}{c}
\sqrt{d} \\
1
\end{array}\right) A(\xi, \tau)+\varepsilon^{2}\left(\begin{array}{c}
X_{02} \\
Y_{02} \\
X_{12} \\
Y_{12} \\
X_{22} \\
Y_{22}
\end{array}\right)+\cdots+\varepsilon^{3}\left(\begin{array}{c}
X_{13} \\
Y_{13}
\end{array}\right)+\right.
$$

Here the $X_{i j}, Y_{i j}$ are functions of $\xi$ and $\tau$ for every $i, j \in \mathrm{N}$. Substituting this expansion into (2.1) and gathering terms of the form $\varepsilon^{a} e^{i k_{c} b x}$ for $a_{1}, b_{1} \in \mathrm{N}$ will lead to the GL-equation. The equations at the $a_{1}=2$-level can be solved: the functions in expansion (3.5) can all be expressed in terms of $A$, the unknown amplitude. The solvability condition for $\left(\begin{array}{c}X_{13} \\ Y_{13}\end{array}\right)$ at the $a_{1}=3, b_{1}=1$-level, yields the GL-equation for $A$ (see Appendix A for the details of the derivation):

$$
A_{\tau}=\frac{v_{1} \sqrt{d}}{(1-d)} A+\frac{4 \sqrt{d}(s-\sqrt{d})}{(1-d)} A_{\xi \xi}+\frac{h}{(1-d)} A|A|^{2}
$$

where

$$
\begin{aligned}
h= & -\left[\sqrt{d}\left(2 \alpha_{1} \sqrt{d}+\alpha_{2}\right)-\left(2 \beta_{1} \sqrt{d}+\beta_{2}\right)\right] F \\
& -\left[\sqrt{d}\left(\sqrt{d} \alpha_{2}+2 \alpha_{3}\right)-\left(\sqrt{d} \beta_{2}+2 \beta_{3}\right)\right] G+3\left(a d^{2}-b\right) \\
F= & -\frac{d}{9(s-\sqrt{d})^{2}}(\alpha(15 s+4 \sqrt{d})-19 \beta) \\
G= & -\frac{1}{9(s-\sqrt{d})^{2}}(19 d \alpha+\beta(15 s-34 \sqrt{d}))
\end{aligned}
$$




$$
\begin{aligned}
& \alpha=d \alpha_{1}+\sqrt{d} \alpha_{2}+\alpha_{3} \\
& \beta=d \beta_{1}+\sqrt{d} \beta_{2}+\beta_{3} .
\end{aligned}
$$

where $\alpha_{1}, \cdots, \beta_{3}$ are introduced by the non-linear terms (3.1) and (3.2). Note that $h, F$ and $G$ seem to blow up as $s \rightarrow s_{e F K}=\sqrt{d}$. We refer to [3] and the references given there for results on the validity of (3.6).

The equation (3.6) can be brought into a standard-form by rescaling $\xi$, $\tau$ and $A$ (introducing $\tilde{\tau}=c_{1} \tau, \tilde{\xi}=c_{2} \xi$ and $A=c_{3} \tilde{A}$ ). We can now choose the $c_{1}, c_{2}$ and $c_{3}$ so that, after omitting the tilde, the equation becomes,

$$
A_{\tau}=A+A_{\xi \xi}+l A|A|^{2}
$$

where $l= \pm 1$. The sign of $l$ depends on the sign of $h$.

Note that there are three situations in which this scaling degenerates. First we note that the diffusion constant $\frac{4 \sqrt{d}(s-\sqrt{d})}{(1-d)}$ in (3.6) disappears as $s \downarrow \sqrt{d}$, the study of this process is the main topic of this paper: it describes the transition of the GL-equation to the eFK-equation. The second bifurcation occurs as $h=0$ (or $|h| \ll 1)$. In this case $(3.6)$ is not the correct modulation equation: it should be replaced by the so-called degenerate GL-equation (see $[9,16]$ and $[27]$ for a validity result). This bifurcation will be encountered in section 4.1. The third degeneration, $d=1$, corresponds to a fundamental observation due to Turing ([29]).

\subsection{The eFK2-equation}

We consider $s$ near $s_{e F K}$ (on $\Gamma_{1}$ or $\Gamma_{2}$ ), i.e. we set $s=s_{e F K}+\sigma \delta$, with $0<\delta \ll 1$. Thus, $k_{c}=0$ and $r_{c}=-\frac{1}{s}=-\frac{1}{\sqrt{d}}+\frac{\sigma \delta}{d}-\frac{\sigma^{2} \delta^{2}}{d \sqrt{d}}+O\left(\delta^{3}\right)$. Note that $\sigma>0$ corresponds to figure $2 \mathrm{c}: \omega_{1}\left(k, r_{c}\right)$ has three extremes that merge as $\sigma \downarrow 0 ; \sigma<0$ is represented by figure $2 \mathrm{~b}$. As above we choose $r O\left(\delta^{2}\right)$ close to $r_{c}: r=r_{c}-v_{2} \delta^{2}$ where $v_{2}>0$. Therefore, by substitution of the expressions for $r_{c}$ and $k_{c}$ into (3.3), it follows in highest order that $\mathcal{M}_{c}$ is the same as defined in section 3.1 and the solvability condition is also as given there. Below we will introduce a number of scalings which are different from those used in the classical GL case. In section 4 we will analyse the transition from the classical GL case (see section 3.1) to this eFK case; there, these scalings will also be explained in more detail.

We model the perturbation of the solution $(0,0)$ as a slow modulation of the 'most unstable wave' with wavenumber $k=k_{c}=0$. However, here this 'wave' $e^{i k_{c} x}\left(\begin{array}{l}. \\ \cdot\end{array}\right)$ reduces to $1\left(\begin{array}{l}\cdot \\ \cdot\end{array}\right)$. This yields that in this case we cannot expand the perturbation as both a Fourier series and a Taylor series in 
$\delta$. Thus, (3.5) has to be replaced by

$$
\left(\begin{array}{l}
u \\
v
\end{array}\right)=\delta^{p}\left(\begin{array}{c}
\sqrt{d} \\
1
\end{array}\right) B\left(\xi_{B}, \tau_{B}\right)+\delta^{p+1}\left(\begin{array}{c}
X_{01} \\
Y_{01}
\end{array}\right)+h o t
$$

where $\left(\begin{array}{c}\sqrt{d} \\ 1\end{array}\right)$ is again the 'most critical' direction. Note that the unknown amplitude $B$ has to be real now: all eigenvalues and eigenfunctions are real. The replacement of the complex amplitude $A$ by a real amplitude $B$ will (also) be discussed in section 4 . In standard non-linear stability analysis, as in the derivation of the GL-equation in section 3.1, the perturbation of the solution $(0,0)$ is taken to be $O(\delta)$ i.e. $p=1$. Using the same assumption in this case and substituting the expansion into the reaction-diffusion system (2.1), leads on the $O\left(\delta^{2}\right)$-level to an inconsistent system of equations: on the $\delta^{2}$-level we obtain

$$
\begin{aligned}
0 & =-\frac{1}{\sqrt{d}} X_{01}+\frac{\sigma}{\sqrt{d}} B+Y_{01}+\alpha B^{2} \\
0 & =\sqrt{d} Y_{01}+\sigma B-X_{01}+\beta B^{2}
\end{aligned}
$$

where $\alpha$ and $\beta$ are as defined in (3.10) and (3.11) in section 3.1. This leads to

$$
\mathcal{M}_{c}\left(\begin{array}{c}
X_{01} \\
Y_{01}
\end{array}\right)=-\sigma\left(\begin{array}{c}
\frac{1}{\sqrt{d}} \\
1
\end{array}\right) B-\left(\begin{array}{c}
\alpha \\
\beta
\end{array}\right) B^{2} .
$$

Applying the solvability condition gives

$$
\left(\alpha-\frac{\beta}{\sqrt{d}}\right) B^{2}=0,
$$

which yields, since $\alpha$ and $\beta$ are arbitrary constants: $B=0$, i.e. we need to consider smaller perturbations. Therefore, we are forced to choose $p=2$ in this subsection. The variables $\xi_{B}$ and $\tau_{B}$ are slow space and time variables, where the scaling of $\tau_{B}$ is standard: $\tau_{B}=\delta^{2} t$. The scaling of $\xi_{B}$ is not the same as in the GL case. It is related to the width of the interval of the $k$-values for which the solution $(0,0)$ is unstable (the largest eigenvalue is positive for these $k$ ) at $r=r_{c}-v_{2} \delta^{2}$ (as is also the case in the derivation of the GL-equation). Therefore we have to determine for which $k$ the eigenvaluecurve of the largest eigenvalue intersects the $k$-axis. Substituting $\omega=0$ and the expressions for $r$ and $s$ into (2.4) leads in highest order to

$$
d k^{4}+2 \delta \sigma k^{2}-v_{2} \sqrt{d} \delta^{2}=0 .
$$

This equation has two solutions: $k= \pm\left(\frac{\sqrt{\sigma^{2}+v_{2} d \sqrt{d}}-\sigma}{d}\right)^{\frac{1}{2}} \sqrt{\delta}$. Therefore the width of the interval of the unstable $k$-values is of order $\sqrt{\delta}$ and we scale 
$\xi_{B}=\sqrt{\delta} x$. The higher order terms in the expansion of the perturbation are modelled as

$$
\left(\begin{array}{l}
u \\
v
\end{array}\right)=\delta^{2}\left(\begin{array}{c}
\sqrt{d} \\
1
\end{array}\right) B\left(\xi_{B}, \tau_{B}\right)+\delta^{3}\left(\begin{array}{c}
X_{01} \\
Y_{01}
\end{array}\right)+\delta^{4}\left(\begin{array}{c}
X_{02} \\
Y_{02}
\end{array}\right)+\text { hot. }
$$

Here the $X_{0 i}, Y_{0 i}$ are real functions of $\xi_{B}$ and $\tau_{B}$ for every $i \in \mathbf{N}, i>0$. Substituting this expansion into (2.1) and sorting together the terms which have the same order of $\delta$ will lead, by applying the solvability condition (3.4), to the eFK2-equation on the $\delta^{4}$-level. On the $\delta^{3}$-level we have

$$
\mathcal{M}_{c}\left(\begin{array}{c}
X_{01} \\
Y_{01}
\end{array}\right)=-\sigma\left(\begin{array}{c}
\frac{1}{\sqrt{d}} \\
1
\end{array}\right) B-\left(\begin{array}{c}
\sqrt{d} \\
d
\end{array}\right) B_{\xi_{B} \xi_{B}} .
$$

Applying the solvability condition (3.4) leads to the trivial condition

$$
-\sigma\left(\frac{1}{\sqrt{d}}-\frac{1}{\sqrt{d}}\right) B-\left(\sqrt{d}-\frac{d}{\sqrt{d}}\right) B_{\xi_{B} \xi_{B}}=0 .
$$

Hence, the inconsistency which appeared for choosing $p=1$ in (3.13), is no longer there. Because $\operatorname{det} \mathcal{M}_{c}=0$, equation (3.17) does not have an unique solution, therefore we have to introduce a second amplitude function $B_{1}$ which depends on $\xi_{B}$ and $\tau_{B}$. Then $X_{01}$ and $Y_{01}$ can be solved in terms of $B$ and $B_{1}$ :

$$
\left(\begin{array}{c}
X_{01} \\
Y_{01}
\end{array}\right)=\left(\begin{array}{c}
\sigma B+d B_{\xi_{B} \xi_{B}} \\
0
\end{array}\right)+\left(\begin{array}{c}
\sqrt{d} \\
1
\end{array}\right) B_{1}\left(\xi_{B}, \tau_{B}\right) .
$$

The equation on the $\delta^{4}$-level reads

$$
\begin{aligned}
\mathcal{M}_{c}\left(\begin{array}{c}
X_{02} \\
Y_{02}
\end{array}\right)= & \left(\begin{array}{c}
\sqrt{d} \\
1
\end{array}\right) B_{\tau_{B}}+\left(\begin{array}{c}
v_{2} \sqrt{d}+\frac{\sigma^{2}}{d} \\
0
\end{array}\right) B-\sigma\left(\begin{array}{c}
\frac{1}{d} X_{01} \\
Y_{01}
\end{array}\right) \\
& -\left(\begin{array}{c}
X_{01 \xi_{B} \xi_{B}} \\
d Y_{01 \xi_{B} \xi_{B}}
\end{array}\right)-\left(\begin{array}{c}
\alpha \\
\beta
\end{array}\right) B^{2}
\end{aligned}
$$

where $\alpha$ and $\beta$ are as in equations (3.10) and (3.11) in section 3.1. Applying the solvability condition (3.4) here yields

$$
\begin{aligned}
(d-1) B_{\tau_{B}} & +\left(v_{2} d+\frac{\sigma^{2}}{\sqrt{d}}\right) B-\sigma\left(\frac{1}{\sqrt{d}} X_{01}-Y_{01}\right) \\
& -\left(\sqrt{d} X_{01 \xi_{B} \xi_{B}}-d Y_{01 \xi_{B} \xi_{B}}\right)-(\sqrt{d} \alpha-\beta) B^{2}=0 .
\end{aligned}
$$

Substituting (3.19) gives

$$
B_{\tau_{B}}=\frac{1}{1-d}\left(v_{2} d B-2 \sqrt{d} \sigma B_{\xi_{B} \xi_{B}}-d \sqrt{d} B_{\xi_{B} \xi_{B} \xi_{B} \xi_{B}}-(\sqrt{d} \alpha-\beta) B^{2}\right)(3.21)
$$


This equation can be brought into a standard-form by rescaling the $\tau_{B}, \xi_{B}$ and $B$

$$
B_{\tau_{B}}=B+D B_{\xi_{B} \xi_{B}}-B_{\xi_{B} \xi_{B} \xi_{B} \xi_{B}}-B^{2},
$$

where $D=D\left(\sigma, v_{2}, d\right)=-\frac{2 \sigma}{\sqrt{v_{2}} d^{\frac{3}{4}}}$ is the only parameter left. Note that the sign of $D$ decides between an eigenvalue-curve with three critical points $(D<0$ or $\sigma>0)$ or one critical point $(D>0, \sigma<0)$. Besides the Turing degeneration at $d=1$ ([29]) we also encounter again a 'non-linear' degeneration as $\sqrt{d} \alpha-\beta=0$, see sections 3.4 and 5.4 .

\subsection{More modulation equations}

So far, we derived on $\Gamma_{2}$ for $s_{e F K}<s<s_{c G L}$, the GL-equation and for $s$ close to $s_{e F K}$, the eFK2-equation. In this section we give the modulation equations which can be derived for other choices of $s$. We will only state the equations here and will not derive or study them any further.

We start on $\Gamma_{1}$ with $s$ not close to $s_{e F K}$, then $k_{c}=0$ and a FisherKolmogorov equation can be derived:

$$
\left(1-s^{2}\right) B_{\tau}=v_{2} s^{2} B-\left(s^{2}-d\right) B_{\xi \xi}-(s \alpha-\beta) B^{2} .
$$

Here $\alpha$ and $\beta$ are as defined in section 3.1 and $\tau=\varepsilon^{2} t$ and $\xi=\varepsilon x$. The perturbation of the solution $(0,0)$ is taken of $O\left(\varepsilon^{2}\right)$, as in the case of the eFK-equation. Of course there is a transition from this FK-equation to the eFK-equation by letting $s \rightarrow s_{e F K}$, but we will not study this transition here.

There is a second branch of GL-bifurcations for $r=-s\left(\right.$ on $\left.\Gamma_{3}\right)$, see figure 3. Here the eigenvalue-curve has one maximum in $k_{c}=0$ and around this maximum the eigenvalues are complex. Therefore a complex GL-equation can be derived where the perturbation of the solution is taken around the 'wave' $e^{i \omega_{c} t}$ where $\omega_{c}=\operatorname{Im} \omega\left(0, r_{c}\right)$ :

$$
C_{\tau}=r_{3} C+d_{3} C_{\xi \xi}+c_{3} C|C|^{2} .
$$

Here $r_{3}, d_{3}$ and $c_{3}$ are complex-valued (see for instance Kuramoto [20] for the derivation of this equation in the Brusselator model and [26] for a validity result). One would expect here that the space variable $\xi$ has to be chosen as a 'travelling' variable, $\xi=\varepsilon(x+\nu t)$ where $\nu=\left.\operatorname{Re} \frac{1}{i} \frac{\partial \omega}{\partial k}\right|_{\left(k_{c}, r_{c}\right)}$, see [14]. However, since $k_{c}=0$, it follows that $\nu=0$ thus the scaling of $\xi$ is as before. The two GL-bifurcation branches $\Gamma_{2}$ and $\Gamma_{3}$ intersect at the co-dimension 2 bifurcation point $\left(r_{c G L}, s_{c G L}\right)$, see figure 3 . Both 'instability mechanisms' (at $k=k_{c}$ and at $k=0$ ) can interact here, thus the bifurcation is described by a system of coupled GL-equations:

$$
\begin{aligned}
& A_{\tau}=r_{1} A+d_{1} A_{\xi \xi}+c_{1} A|A|^{2}+c_{2} A|C|^{2} \\
& C_{\tau}=r_{2} C+d_{2} C_{\xi \xi}+c_{3} C|C|^{2}+c_{4} C|A|^{2} .
\end{aligned}
$$


Again the $\xi$-variable is as before. By setting $C=0$ we recover the real GL-

equation described in section 3.1 around the critical wave $e^{i k_{c} x}, k_{c}=\sqrt{\frac{s-\sqrt{d}}{d}}$ (thus $r_{1}, d_{1}, c_{1} \in \mathbf{R}$ ); $A=0$ yields the above described complex GL-equation for $C$. We refer to [11] for more information (general derivation, behaviour of solutions, references) on coupled GL-equations.

\subsection{The eFK-equation with cubic terms}

Up till now we studied the reaction-diffusion system (2.1) with general nonlinear terms $N_{1}$ and $N_{2}$ as given in (3.1) and (3.2). As we showed in section 3.2 , this leads for $s$ close to $s_{e F K}$ to an eFK-equation with quadratic nonlinear terms. We can also study the case when $N_{1}$ and $N_{2}$ do not contain quadratic terms but only cubic terms. We will show that in this case the behaviour of 'patterns' near $\left(r_{e F K}, s_{e F K}\right)$ is governed by an eFK-equation with cubic terms (denoted by eFK3). This observation is especially interesting, since the eFK-equation with cubic terms is studied extensively in literature (see $[24,8,18]$ and the references given there). See also section 5.4 for a discussion. Thus we consider in (2.1) non-linear terms of the following form:

$$
N_{1}(u, v)=-a u^{3}, \quad N_{2}(u, v)=-b v^{3},
$$

which follows from setting $\alpha_{i}=\beta_{i}=0$ for $i=1,2,3$ in (3.1) and (3.2). We again set $s=s_{e F K}+\sigma \delta$, with $k_{c}, r_{c}$ and $r$ as defined in section 3.2. We model the perturbation of the solution $(0,0)$ as a slow modulation of the wave with wavenumber $k=k_{c}=0$. We now note that the inconsistency which appears in section 3.2 if we consider $p=1$ does not appear: (3.15) is again 'trivial' since $\alpha=\beta=0$ here. Thus, we expand

$$
\left(\begin{array}{l}
u \\
v
\end{array}\right)=\delta\left(\begin{array}{c}
\sqrt{d} \\
1
\end{array}\right) B\left(\xi_{B}, \tau_{B}\right)+h o t,
$$

where $B$ is a real amplitude function which depends on the slow space and time variables $\xi_{B}$ and $\tau_{B}$, which were defined in section 3.2. The scaling of $\tau_{B}$ is standard: $\tau_{B}=\delta^{2} t$ and since the eigenvalue-curve is still the same as in section 3.2, the scaling of the $\xi_{B}$ is the same as in that section: $\xi_{B}=\sqrt{\delta} x$. The higher order terms in the expansion are given as

$$
\left(\begin{array}{c}
u \\
v
\end{array}\right)=\delta\left(\begin{array}{c}
\sqrt{d} \\
1
\end{array}\right) B\left(\xi_{B}, \tau_{B}\right)+\delta^{2}\left(\begin{array}{c}
X_{01} \\
Y_{01}
\end{array}\right)+\delta^{3}\left(\begin{array}{c}
X_{02} \\
Y_{02}
\end{array}\right)+\text { hot. }
$$

where the $X_{0 i}, Y_{0 i}$ are real functions of $\xi_{B}$ and $\tau_{B}$ for every $i \in \mathrm{N}, i>0$. After substituting this expansion into (2.1) and solving the equations on the $\delta^{2}$-level (as in section 3.2 ), we find the eFK3-equation by the application of the solvability condition on the $\delta^{3}$-level:

$$
B_{\tau_{B}}=\frac{v_{2} d}{(1-d)} B-\frac{2 \sqrt{d} \sigma}{(1-d)} B_{\xi_{B} \xi_{B}}-\frac{d \sqrt{d}}{(1-d)} B_{\xi_{B} \xi_{B} \xi_{B} \xi_{B}}+\frac{\left(a d^{2}-b\right)}{(1-d)} B^{3}(3.26)
$$


This equation can again be brought into standard-form by rescaling the $\tau_{B}, \xi_{B}$ and $B$ :

$$
B_{\tau_{B}}=B+D B_{\xi_{B} \xi_{B}}-B_{\xi_{B} \xi_{B} \xi_{B} \xi_{B}}+l B^{3},
$$

Here $l= \pm 1$ and $D$ is as in section 3.2, the sign of $l$ depends on the sign of $a d^{2}-b$. Note that in rescaling the eFK-equation with quadratic terms the sign of the non-linear term was irrelevant but now in the case of the eFK3equation, the sign of $a d^{2}-b$ is very important in reducing the equation to standard-form: as in the GL case it is important for the existence of bounded solutions.

\section{The transition from the GL-equation to the eFK2- equation}

In section 3.1 we described the non-linear evolution of small solutions of (2.1) by a GL-equation, since $s>s_{e F K}$ and $\left|s-s_{e F K}\right|=O(1)$. We found in section 3.2 that the evolution of small solutions is described by the eFK2equation when $\left|s-s_{e F K}\right|=O(\delta) \ll 1$. In this section we will study the transition between these two modulation equations.

First we will study the GL-equation for $\left|s-s_{e F K}\right|=O(\delta) \ll 1$ : in this case one can still derive the GL-equation as long as $r-r_{c}=-v_{1} \varepsilon^{2} \ll O\left(\delta^{2}\right)$. At leading order this GL-equation is exactly the same as the one derived in section 3.1, however, now the higher order derivatives $A_{\xi \xi \xi}$ and $A_{\xi \xi \xi \xi}$ are of order $O\left(\frac{\varepsilon}{\delta}\right)$, respectively $O\left(\frac{\varepsilon^{2}}{\delta^{2}}\right)$, and thus much larger than in section 3.1 . Also, we will find that, the coefficient $h$ of the non-linear term becomes positive.

Thus, in the overlap region $\left|s-s_{e F K}\right|=O(\delta) \ll 1$ both modulation equations, the eFK2-equation and the singularly perturbed GL-equation, describe the evolution of small solutions. However, these equations describe different processes, as we shall show in detail below. At this point this can be seen by noticing that the eFK2-equation governs the evolution of $O\left(\delta^{2}\right)$ solutions on the time scale $\tau_{B}=\delta^{2} t$ and the spatial scale $\xi_{B}=\sqrt{\delta} x$ (see section 3.2 ), while we shall find in section 4.1 that the singularly perturbed GL-equation governs $O(\varepsilon \delta)$ solutions on a $O\left(\frac{1}{\varepsilon^{2}}\right)$ time scale and a $O\left(\frac{\varepsilon}{\sqrt{\delta}}\right)$ spatial scale. Observe that these scalings merge as $\varepsilon \rightarrow \delta$, but, we shall see that in this limit the derivation process leading to the singularly perturbed GL-equation looses its validity. However, we will show in section 4.1 that in this limit, the GL-equation can be interpreted as an equation governing the evolution of a special class of spatially periodic solutions of the eFK2equation. This interpretation, for instance, enables us to understand the 
relation between the quadratic non-linear term in the real eFK2-equation and the cubic non-linear term in the complex GL-equation.

First we study the form of the eigenvalue-curve for $s=s_{e F K}+\delta$, see also figure $2 \mathrm{c}$. We find that the difference in the eigenvalue between the maxima and the minimum of the curve is of $O\left(\delta^{2}\right)$. This can be seen as follows: at $r=r_{c}=\frac{s}{d}-\frac{2}{\sqrt{d}}$ we have that $\omega\left(k_{c}, r_{c}\right)=0$ and $\omega\left(0, r_{c}\right)=$ $\frac{1}{2}\left(s+r_{c}+\sqrt{\left(s-r_{c}\right)^{2}-4}\right)$. Substituting the expressions for $r_{c}$ and $s$ gives that $\omega\left(0, r_{c}\right)=O\left(\delta^{2}\right)$ and thus the difference between $\omega\left(k_{c}, r\right)$ and $\omega(0, r)$ is of order $\delta^{2}$.

\subsection{The singularly perturbed GL-equation}

Now we study the derivation of the modulation equation for $\delta \gg \varepsilon$, this is quite similar to the derivation of the GL-equation in section 3.1. We set $s$ as in section 3.2 with $\sigma=1$, thus $s=s_{e F K}+\delta$. Here $r_{c}=-\frac{1}{\sqrt{d}}+\frac{\delta}{d}, k_{c}^{2}=\frac{\delta}{d}$ and we assume $r$ to be close to $r_{c}: r=r_{c}-v_{1} \varepsilon^{2}$ with $v_{1}>0$. One of the differences with the GL derivation in section 3.1 is the order of magnitude of the perturbations of the trivial solution $(0,0)$. Taking the perturbation of $O(\varepsilon)$ leads to an inconsistent system on the $O\left(\varepsilon^{2}\right)$-level, which appears in the same way as we showed in section 3.2, therefore we must take the perturbation of order $\varepsilon \delta$ :

$$
\left(\begin{array}{l}
u \\
v
\end{array}\right)=\varepsilon \delta\left(\begin{array}{c}
\sqrt{d} \\
1
\end{array}\right) A\left(\xi_{A}, \tau_{A}\right) e^{i k_{c} x}+\text { c.c. }+ \text { hot. }
$$

Here $\tau_{A}$ and $\xi_{A}$ are slow time and space variables, with for $\tau_{A}$ the standard scaling $\tau_{A}=\varepsilon^{2} t$. The scaling of $\xi_{A}$ is again related to the width of the interval of the $k$-values at which the solution $(0,0)$ is unstable. Thus we have to determine for which $k$-values $\omega_{1}=0$. Substituting $\omega=0$ and the expressions for $r$ and $s$ into (2.4) leads in highest order to $k^{2}=\frac{\delta}{d}\left(1 \pm \frac{\sqrt{v_{1}} d^{\frac{3}{4}}}{\delta} \varepsilon\right)$ which gives four solutions: $k= \pm k_{c} \pm O\left(\frac{\varepsilon}{\sqrt{\delta}}\right)$. Therefore the width of the interval of unstable $k$-values is of $O\left(\frac{\varepsilon}{\sqrt{\delta}}\right)$ and we scale $\xi_{A}=\frac{\varepsilon}{\sqrt{\delta}} x$.

It is a priori not clear how to choose the magnitudes of the harmonics and the higher order terms of (4.1). For instance, the non-linear interactions suggest that the $e^{2 i k_{c} x}$ and the $e^{0 x}$ mode should be $O\left(\varepsilon^{2} \delta^{2}\right)$, while

$$
\frac{\partial^{2}}{\partial x^{2}}\left(\varepsilon \delta A e^{i k_{c} x}\right)=\varepsilon\left(\varepsilon^{2} A_{\xi_{A} \xi_{A}}+2 i \frac{\varepsilon \delta}{\sqrt{d}} A_{\xi_{A}}-\delta k_{c}^{2} A\right) e^{i k_{c} x},
$$

which suggests that a higher order correction on the $e^{i k_{c} x}$ mode should become $O\left(\varepsilon^{2} \delta\right)$. However, it follows from the computations in the appendix (see equations (A.1), (A.2)) that the $\left(\begin{array}{c}X_{02} \\ Y_{02}\end{array}\right)$ and $\left(\begin{array}{c}X_{22} \\ Y_{22}\end{array}\right)$ vectors in 
do not remain $O(1)$ as $s=\sqrt{d}+\delta$. Thus, the higher order terms of (4.1) should be modelled as:

$$
\begin{gathered}
\varepsilon^{2}\left(\begin{array}{c}
X_{02} \\
Y_{02}
\end{array}\right)+\cdots \\
\left(\begin{array}{c}
u \\
v
\end{array}\right)=e^{i k_{c} x}\left(\varepsilon \delta\left(\begin{array}{c}
\sqrt{d} \\
1
\end{array}\right) A+\varepsilon^{2} \delta\left(\begin{array}{c}
X_{12} \\
Y_{12}
\end{array}\right)+\varepsilon^{3}\left(\begin{array}{c}
X_{13} \\
Y_{13}
\end{array}\right)+\right. \\
e^{2 i k_{c} x}\left(\varepsilon^{2}\left(\begin{array}{c}
X_{22} \\
Y_{22}
\end{array}\right)+\cdots+\right.\text { c.c. }
\end{gathered}
$$

Again, $A, X_{i j}, Y_{i j}$ are functions of $\xi_{A}$ and $\tau_{A}$ for every $i, j \in \mathrm{N}$. Substituting this expansion into (2.1) and gathering terms of the form $\varepsilon^{a_{1}} \delta^{a_{2}} e^{i k_{c} b x}$ for $a_{1}, a_{2}, b_{1} \in \mathrm{N}$ will lead to a modulation equation on the $a_{1}=3, a_{2}=1, b_{1}=$ 1-level. The equations on the $\left(a_{1}, a_{2}\right)$-level where $a_{1}+a_{2} \leq 3$ can be solved in terms of $A$. The equation including higher order terms becomes

$$
\begin{aligned}
(1-d) A_{\tau_{A}}= & v_{1} d A+4 \sqrt{d} A_{\xi_{A} \xi_{A}}+h A|A|^{2}-\frac{\varepsilon}{\delta} 4 i d A_{\xi_{A} \xi_{A} \xi_{A}} \\
& -\frac{\varepsilon^{2}}{\delta^{2}} d \sqrt{d} A_{\xi_{A} \xi_{A} \xi_{A} \xi_{A}}+O(\varepsilon),
\end{aligned}
$$

where the $O(\varepsilon)$ terms are the usual higher order terms in the GL-equation (see for instance [9]). Note that the $A_{\xi_{A} \xi_{A} \xi_{A} \xi_{A}}$-term should be included in the $O(\varepsilon)$ terms if $\delta^{2} \geq \varepsilon$. The expression for the coefficient $h$ of the nonlinear term simplifies considerably due to the new scalings and the fact that $s=s_{e F K}+\delta($ see $(3.7)-(3.11))$ :

$$
h=\frac{38}{9} \sqrt{d}(\sqrt{d} \alpha-\beta)^{2}+O(\delta) .
$$

Thus, $h$ is always positive near the eFK-bifurcation! In other words: even if $h<0$ for $s$ not close to $s_{e F K}, h$ will become positive if $s$ decreases towards $s_{e F K}$. This means that there must be a value of $s, s_{n l}$, at which $h$ changes sign. Near $s_{n l}$ the GL-equation should be replaced by a degenerate GLequation (see $[9,16,27]$ ) of the form

$$
A_{\tau}=r A+A_{\xi \xi}-c_{1} A|A|^{2}+c_{2} A|A|^{5}+i\left(c_{3}|A|^{2} A_{\xi}+c_{4} A^{2} \bar{A}_{\xi}\right) .
$$

This behaviour has a drastic effect on the patterns exhibited by (2.1): if $s>s_{n l}$ the GL-approximation predicts stable, stationary, periodic patterns of the form $R e^{i K \xi}$ in (3.6). However, these solutions do not exist for $h>0$. In section 5 we will encounter this phenomenon numerically. In [9] the stability of the stationary periodic patterns to (4.5) is studied.

Note also that the cubic coefficients in the non-linear terms of (2.1) do not have a leading order influence on $h$ as $\left|s-s_{E F K}\right| \ll 1$. Thus, this procedure 
degenerates if we only consider cubic (and higher order) terms in (2.1). This is in agreement with the analysis of section 4: in this case the GL-equation limits on the eFK3-equation (and many scalings are different). In this paper we do not pay attention to the details of this transition: it is quite similar to, and even a bit less complex than, the transition from the GL-equation to the eFK2-equation.

\subsection{The limit $\delta \downarrow O(\varepsilon)$}

The transition case $\varepsilon=O(\delta)$ or $\delta=O(\varepsilon)$, can be obtained in two different ways: either one fixes $r-r_{c}$, i.e. $\varepsilon$, and decreases $s$ (i.e. $\delta$ ), or one fixes $s-s_{e F K}(\delta)$ and increases $r-r_{c}(\varepsilon)$. The behaviour at $\varepsilon=O(\delta)$ is independent of this. Here we consider $\delta \downarrow O(\varepsilon)$. The best way to understand what happens to the GL-equation in this limit, is to interpret the GL Ansatz and scalings in Fourier space. The Fourier transform of the classical decomposition (3.5) (with the scaling $\xi=\varepsilon x$ ) consists of 'peaks' of width $O(\varepsilon)$ around the points $N k_{c}, N \in \mathrm{Z}$; the peaks around $\pm k_{c}$ are of height $O\left(\frac{1}{\varepsilon}\right)$ and the peaks around $\pm 2 k_{c}$ and 0 are $O(1)$. In general, the peaks around $N k_{c}$ are of a $O\left(\varepsilon^{|N|-2}\right)$ height $(N \neq 0)$, see [17] for more details. The Fourier transform of the decomposition leading to the singularly perturbed GL-equation has a similar structure: there are peaks of height $\frac{1}{\varepsilon \delta}$ around $k= \pm k_{c}$ and peaks around $k=0, \pm 2 k_{c}$ of height 1 . All these peaks have a width of $O\left(\frac{\varepsilon}{\sqrt{\delta}}\right)$, see figure 4 . As $\delta$ decreases we see that the width of the peaks increases, while the distance between the peaks, $k_{c}$, decreases (since $k_{c}=O(\sqrt{\delta})$ ). When $\delta$ has become $O(\varepsilon)$ we see that we cannot distinguish between separate peaks: they are all overlapping (see figure 5).

Thus, one cannot assume any longer a decomposition like (4.2). It must be replaced by the eFK decomposition (3.16), see again figure 5: the structure in Fourier space is now only one 'wide' peak of height $\frac{1}{\varepsilon^{2}}$ and width $\sqrt{\varepsilon}$. Note that one can also observe the evolution of the GL Ansatz (4.2) to the eFK Ansatz (3.16) in (4.2) itself: as $\delta$ decreases to $\varepsilon$, the leading order term becomes

$$
\begin{aligned}
\left(\begin{array}{l}
u \\
v
\end{array}\right) & =\varepsilon^{2}\left[\left(\begin{array}{c}
\sqrt{d} \\
1
\end{array}\right) A\left(\xi_{A}, \tau_{A}\right) e^{i \frac{1}{\sqrt{d}} \xi_{B}}+\left(\begin{array}{c}
X_{02} \\
Y_{02}
\end{array}\right)+\left(\begin{array}{c}
X_{22} \\
Y_{22}
\end{array}\right) e^{2 i \frac{1}{\sqrt{d}} \xi_{B}}+\cdots\right] \\
& =\varepsilon^{2} B\left(\xi_{B}, \tau_{B}\right)
\end{aligned}
$$

by definition (note that $k_{c} x=\frac{\delta}{\sqrt{d} \varepsilon} \xi_{A}=\frac{1}{\sqrt{d}} \xi_{B}$ ). Another way to see that the GL Ansatz is not valid anymore is the fact that when $\delta \rightarrow \varepsilon$ all the terms of the form $\frac{\partial^{N}}{\partial \xi^{N}} A$ (for every $N$ ) become $O(1$ ) in equation (4.3)

We now want to study how the transition from the eFK2-equation (3.21) 
to the singularly perturbed GL-equation (4.3) takes place quantitatively. Note that this means that all the coefficients in the equations have to agree. Since $r$ is differently defined in the derivations of the singularly perturbed GL-equation and of the eFK2-equation, the coefficients cannot agree yet. We have taken in the derivations $r_{G L}=r_{c_{G L}}-v_{1} \varepsilon^{2}=-\frac{1}{\sqrt{d}}+\frac{\delta}{d}-v_{1} \varepsilon^{2}$ and $r_{e F K}=r_{c_{e F K}}-v_{2} \delta^{2}=-\frac{1}{\sqrt{d}}+\frac{\delta}{d}-\frac{\delta^{2}}{d \sqrt{d}}-v_{2} \delta^{2}$ (when taking $\sigma=1$ ). Comparing these two expressions for $r$ gives a relation between $v_{1}$ and $v_{2}$ :

$$
v_{2}=-\frac{1}{d \sqrt{d}}+v_{1} \frac{\varepsilon^{2}}{\delta^{2}}
$$

We need to compare the two expansions of the perturbation around the solution $(0,0)$ and equate $(3.16)$ and (4.2). This leads to

$$
\delta^{2} B\left(\xi_{B}, \tau_{B}\right)\left(\begin{array}{c}
\sqrt{d} \\
1
\end{array}\right)+h o t=\varepsilon \delta A\left(\xi_{A}, \tau_{A}\right)\left(\begin{array}{c}
\sqrt{d} \\
1
\end{array}\right) e^{i k_{c} x}+c . c .+ \text { hot }
$$

where $\xi_{A}=\frac{\varepsilon}{\sqrt{\delta}} x, \xi_{B}=\sqrt{\delta} x, \tau_{A}=\varepsilon^{2} t, \tau_{B}=\delta^{2} t$ and $k_{c}=\sqrt{\frac{\delta}{d}}$. Thus

$$
B\left(\xi_{B}, \tau_{B}\right)=\frac{\varepsilon}{\delta} A\left(\xi_{A}, \tau_{A}\right) e^{i \frac{\xi_{B}}{\sqrt{d}}}+\frac{\varepsilon}{\delta} \bar{A}\left(\xi_{A}, \tau_{A}\right) e^{-i \frac{\xi_{B}}{\sqrt{d}}}+h o t
$$

However, the higher order terms cannot all be neglected, as we will see below. Therefore, we write

$$
B\left(\xi_{B}, \tau_{B}\right)=\frac{\varepsilon}{\delta} \sum_{n=-\infty}^{\infty} \psi_{n}\left(\xi_{A}, \tau_{A}\right) e^{i n \frac{\xi_{B}}{\sqrt{d}}}
$$

where $\psi_{-n}=\bar{\psi}_{n}$ since $B$ is real (the $\psi_{n}$ 's, $n \neq 1$, correspond to the $\left(\begin{array}{c}X_{n 2} \\ Y_{n 2}\end{array}\right)$ amplitude of the harmonics in (4.2)). We know that $\psi_{1}=A$ and that $\left|\psi_{n}\right| \ll 1$ for $n \neq 1$. Substituting this expression for $B$ into the eFK2equation (3.21), we find, after multiplying by $\frac{\delta}{\varepsilon}$, the following set of equations for the $\psi_{n}$ :

$$
\begin{aligned}
\frac{\varepsilon^{2}}{\delta^{2}}(1-d) \psi_{n_{\tau_{A}}}= & \left(-\frac{\left(n^{2}-1\right)^{2}}{\sqrt{d}}+v_{1} d \frac{\varepsilon^{2}}{\delta^{2}}\right) \psi_{n}+\frac{4 i n \varepsilon}{\delta}\left(n^{2}-1\right) \psi_{n_{\xi_{A}}} \\
& +2 \sqrt{d}\left(3 n^{2}-1\right) \frac{\varepsilon^{2}}{\delta^{2}} \psi_{n_{\xi_{A} \xi_{A}}}-4 i n d \frac{\varepsilon^{3}}{\delta^{3}} \psi_{n_{\xi_{A} \xi_{A} \xi_{A}}} \\
& -d \sqrt{d} \frac{\varepsilon^{4}}{\delta^{4}} \psi_{n_{\xi_{A} \xi_{A} \xi_{A} \xi_{A}}}-\frac{\varepsilon}{\delta}(\sqrt{d} \alpha-\beta) \sum_{l=-\infty}^{\infty} \psi_{l} \psi_{n-l} .
\end{aligned}
$$

where we have already used (4.6). Now, we want to derive the equation for $\psi_{n}(=A)$ : we should obtain the singularly perturbed GL-equation. Recall 
that $\left|\psi_{n}\right| \ll 1$ for $n \neq 1$ which implies that the $\psi_{n}$ for $n \neq \pm 1$ still can be rescaled. For $n=0$ we obtain, at leading order,

$$
0=-\frac{1}{\sqrt{d}} \psi_{0}-2 \frac{\varepsilon}{\delta}(\sqrt{d} \alpha-\beta) \sum_{l=0}^{\infty}\left|\psi_{l}\right|^{2}
$$

This implies that we must rescale $\psi_{0}$ with the factor $\frac{\varepsilon}{\delta}(\ll 1$ when $\varepsilon \ll \delta)$ to obtain a consistent system. Thus we introduce $\psi_{0}=\frac{\varepsilon}{\delta} \tilde{\psi}_{0}$. This yields

$$
\tilde{\psi}_{0}=-2 \sqrt{d}(\sqrt{d} \alpha-\beta)\left|\psi_{1}\right|^{2} .
$$

Setting $n=2$ gives, at leading order,

$$
0=-\frac{9}{\sqrt{d}} \psi_{2}-\frac{\varepsilon}{\delta}(\sqrt{d} \alpha-\beta) \sum_{l=-\infty}^{\infty} \psi_{l} \psi_{2-l},
$$

which gives us that $\psi_{2}$ also must be scaled with $\frac{\varepsilon}{\delta}$, therefore we introduce $\psi_{2}=\frac{\varepsilon}{\delta} \tilde{\psi}_{2}$. And obtain

$$
\tilde{\psi}_{2}=-\frac{\sqrt{d}}{9}(\sqrt{d} \alpha-\beta) \psi_{1}^{2}
$$

The equation for $\psi_{1}$ reads:

$$
\begin{aligned}
(1-d) \psi_{1_{\tau_{A}}}= & v_{1} d \psi_{1}+4 \sqrt{d} \psi_{1_{\xi_{A} \xi_{A}}}-\frac{\varepsilon}{\delta} 4 i d \psi_{1_{\xi_{A} \xi_{A} \xi_{A}}}-\frac{\varepsilon^{2}}{\delta^{2}} d \sqrt{d} \psi_{1_{\xi_{A} \xi_{A} \xi_{A} \xi_{A}}} \\
& -2(\sqrt{d} \alpha-\beta)\left(\tilde{\psi}_{2} \bar{\psi}_{1}+\tilde{\psi}_{0} \psi_{1}\right)+\text { hot. }
\end{aligned}
$$

Substituting the expressions (4.9) and (4.10) for $\tilde{\psi}_{0}$ and $\tilde{\psi}_{2}$, finally gives exactly the singularly perturbed GL-equation (4.3) for $\psi_{1}$.

Thus in the region $0<\varepsilon \ll \delta \ll 1$ one can derive the GL-equation from the eFK2-equation by using the relation (4.7). However, one should be careful here: inserting (4.7) violates the assumptions made in the derivation of the eFK2-equation (section 3.2). There, we assume that $B=O(1)$ and that it is a function of the spatial scale $\xi_{B}$ and time scale $\tau_{B}$. This is clearly not the case in (4.7). Another way to see this is to look once again at figure 4 : here we plotted both the singularly perturbed GL-peaks and the eFK-peaks for $0<\varepsilon \ll \delta \ll 1$. These structures describe different phenomena. One cannot describe the GL behaviour using the eFK Ansatz. Thus, the singularly perturbed GL-equation and the eFK2-equation do not coincide in the overlap region $0<\varepsilon \ll \delta \ll 1$ (they coincide as $\delta=O(\varepsilon)$ but then the GL-equation is no longer valid). However, the above derivation of an equation for $\psi_{1}=A$ is still useful: it clearly shows, for instance, the subtle relation between the quadratic non-linearity in the eFK2-equation and the cubic non-linearity in the GL-equation. 
Remark 4.1 We can now compare the $\tilde{\psi}_{0}$ and $\tilde{\psi}_{2}$, which are given above, with the $\left(\begin{array}{c}X_{02} \\ Y_{02}\end{array}\right)$ and $\left(\begin{array}{c}X_{22} \\ Y_{22}\end{array}\right)$ appearing in the derivation of the GLequation, see section 3.1 and Appendix A. Setting $s=\sqrt{d}+\delta$ in equation (A.1) gives, after rescaling $A$ with $\delta$, for $Y_{02}$ exactly the same equation as we obtained for $\tilde{\psi}_{0}$. In the same way, after rescaling $A$ with $\delta$ in equation (A.2), we obtain for $Y_{22}$ the same equation as for $\tilde{\psi}_{2}$. This rescaling of $A$ comes from the fact that the first order term of both expansions of the perturbations (for the GL-equation in section 3.1 and for the singularly perturbed GL-equation in section 4.1) differ by a factor $\delta$.

\subsection{Deriving the GL-equation within the eFK2-equation}

If one studies the eFK2-equation on an unbounded domain, one can analyse the non-linear stability of the trivial (i.e. $\equiv 0$ ) solution by the 'GL approach' of section 3.1. Below we show that we can derive the GL-equation within the eFK2-equation. Note that this has also been done rigorously in [5] for the $\mathrm{SH}$ (or eFK3)-equation. Here we present the asymptotic approach to relate it to the transition studied in section 4.2. Thus we study the eFK2-equation (3.21) and write it as

$$
B_{\tau_{B}}=L(B)+N(B)
$$

where

$$
\begin{aligned}
L & =\frac{1}{1-d}\left(d v_{2}-2 \sqrt{d} \sigma \frac{\partial^{2}}{\partial \xi_{B}^{2}}-d \sqrt{d} \frac{\partial^{4}}{\partial \xi_{B}^{4}}\right) \\
N(B) & =-\frac{(\sqrt{d} \alpha-\beta)}{1-d} B^{2} .
\end{aligned}
$$

This system has a basic solution $B \equiv 0$. The linearised stability of the solution is determined by setting

$$
B(\xi, \tau)=c e^{\tau \omega\left(k, v_{2}\right)+i k \xi}+c . c .
$$

Substituting this into the linear part of the equation gives

$$
\omega\left(k, v_{2}\right)=\frac{1}{1-d}\left(d v_{2}+2 \sqrt{d} \sigma k^{2}-d \sqrt{d} k^{4}\right) .
$$

This gives the eigenvalue-curve. As long as $\omega\left(k, v_{2}\right)<0$ for every $k$, the solution $B=0$ is stable and for $\omega\left(k, v_{2}\right)=0$, the solution becomes unstable. For $\sigma<0$, the eigenvalue-curve has one maximum at $k=0$, we will not study this. For $\sigma>0$, the eigenvalue-curve has two maxima at $k= \pm \sqrt{\frac{\sigma}{d}}$ and one minimum at $k=0$. For $v_{2}<-\frac{\sigma^{2}}{d \sqrt{d}}$ the eigenvalue is negative for all $k$, the solution $B=0$ is stable, whereas for $v_{2}=-\frac{\sigma^{2}}{d \sqrt{d}}$ the solution 
becomes unstable at $k= \pm \sqrt{\frac{\sigma}{d}}$. And so for $v_{2}>-\frac{\sigma^{2}}{d \sqrt{d}}$ there is a whole interval of $k$-values around $k= \pm \sqrt{\frac{\sigma}{d}}$ where the solution is unstable. Thus we can derive a classical modulation equation by the GL approach. Here the critical point is $\left(k_{c}, v_{2_{c r i t}}\right)=\left(\sqrt{\frac{\sigma}{d}},-\frac{\sigma^{2}}{d \sqrt{d}}\right)$. And we take $v_{2}=-\frac{\sigma^{2}}{d \sqrt{d}}+w \mu^{2}$ where $0<\mu \ll 1$ and $w>0$. The perturbation of the basic solution $B=0$ is taken as a slow modulation of the most unstable wave $e^{i k_{c} x}$ :

$$
B(\xi, \tau)=\mu A(\eta, \nu) e^{i k_{c} x}+\text { c.c. }+ \text { hot }
$$

where $\eta$ and $\nu$ are slow space and time variables which are given by $\eta=\mu \xi$ and $\nu=\mu^{2} \tau$ (the standard GL-scaling). Then we can derive on the $\mu^{3} e^{i k_{c} x}$ level the following GL-equation

$$
(1-d) A \tau=w A+4 \sqrt{d} \sigma A_{\eta \eta}+\frac{38}{9} \sqrt{d} \frac{(\sqrt{d} \alpha-\beta)^{2}}{\sigma^{2}} A|A|^{2} .
$$

Note that the Landau-constant is always positive! This equation is exactly the same as the leading order part of the singularly perturbed GL-equation, when we set $\sigma=1$, derived in section 4.1. This is no surprise: the above analysis is in essence the same as that of section 4.2, compare (4.7) to (4.11). Note that $\mu \ll 1$ plays the role of $\frac{\varepsilon}{\delta}$ in section 4.2 .

Thus, the leading order part of the singularly perturbed GL-equation can be considered as a GL-equation within the eFK2-equation. However, as we explained in section 4.2 and figure 4 , this does not mean that the GL-equation can be replaced by the eFK2-equation.

\section{$5 \quad$ Numerical simulations}

In this section, we study numerically the dynamics of the reaction-diffusion system (2.1). For the GL-equation it is proved theoretically that when the Landau-constant is negative there exist, for some interval of wave numbers, stable, periodic solutions, see Appendix B. We will use the existence of these solutions of the GL-equation to look for (periodic) solutions near the eFK-bifurcation.

First we will numerically check the existence of the periodic solutions which are theoretically known to be solutions of the GL-equation. Then we will decrease $s$ to study how the transition to the eFK2-equation influences the behaviour of these solutions. To be able to do numerical simulations we have to restrict $x$ to a bounded interval. This interval has to be large enough to ensure that the boundaries do not influence the dynamics (too much). We refer to section 5.4 for a discussion. We fixed the length of the interval on $x \in[0,400]$. This choice is 'justified' by a numerical check of the theoretical 
predictions of the stability of periodic solutions of the GL-equation.

Most of the parameters in system (2.1) remain fixed during the simulations. We chose for most of the simulations $d=\frac{1}{2}$ and $\alpha_{1}=\alpha_{3}=\beta_{1}=\beta_{3}=$ $0, \alpha_{2}=0.71, \beta_{2}=0.5$ and $a=1, b=3$. We also did simulations for other choices of $\alpha_{1}, \cdots, \beta_{3}, a$ and $b$ and found that the results of the numerical simulations do not really depend on the choice of these parameters (see also section 5.4). The choice of $d$ is so that $d<1$ is satisfied (section 2 ), and it gives us that $s_{e F K}=\frac{1}{2} \sqrt{2} \approx 0.707$ and $s_{c G L}=\frac{2}{3} \sqrt{2} \approx 0.943$ (section 2 ). With above chosen coefficients the Landau-constant (3.7) remains negative for almost all $s$-values. For $s_{e F K}<s \leq 0.709$ it is positive. We did change $s$ and $r$ and the initial conditions, we will explain the choice of initial data in more detail later on.

We used a moving-grid code to integrate system (2.1) which is described in detail in [1], see also [10] for an application to reaction-diffusion systems. The space variable $x$ in system $(2.1)$ is scaled to $\tilde{x}$ so that the numerical simulations take place on the $\tilde{x}$-interval $[0,1]$. We take homogeneous Neumann boundary-conditions:

$$
\frac{\partial u}{\partial \tilde{x}}(\tilde{x}=0, t)=\frac{\partial u}{\partial \tilde{x}}(\tilde{x}=1, t)=\frac{\partial v}{\partial \tilde{x}}(\tilde{x}=0, t)=\frac{\partial v}{\partial \tilde{x}}(\tilde{x}=1, t)=0 .
$$

Because it is known that the GL-equation has stable stationary periodic solutions, the initial conditions are also taken periodic with respect to $x$ :

$$
u(\tilde{x}, 0)=v(\tilde{x}, 0)=0.05 \cos (p \pi x) .
$$

We chose the amplitude of this initial condition to be of $O\left(\varepsilon^{2}\right)$ (we set $\varepsilon^{2}=0.01$ ) because near the eFK-bifurcation we find that the magnitude of the solution is of $O\left(\varepsilon^{2}\right)$, see sections 3.2 and 4.2. In some of the simulations we fixed $p$ to obtain a certain number of periods in the interval $[0,1]$, in other simulations we changed $p$ as to vary the number of periods in the interval. Throughout the simulations we have been looking for asymptotically stable solutions, we only found stable stationary patterns.

\subsection{Checking the GL-equation}

We started the numerical simulations by checking the theoretical results which are known for the GL-equation. Thus we chose $s_{e F K}<s<s_{c G L}$ along $\Gamma_{2}$ (figure 3 ) to be in the interval of $s$-values for which the GL-equation is derived.

First we took as initial data

$$
u(\tilde{x}, 0)=v(\tilde{x}, 0)=0.05 \cos \left(k_{c} x\right),
$$


where $k_{c}$ is the critical $k$-value $\sqrt{\frac{s-\sqrt{d}}{d}}$ (section 2 ) and chose $s$ such that the initial data satisfy the boundary conditions. It follows from GL-theory that this solution is stable (as long as the Landau-constant is negative). Therefore, we calculated for several $s_{E F K}<s<s_{C G L}$ the corresponding $k_{c}$ and $r_{c}$. Throughout the simulations we set $r=r_{c}-\varepsilon^{2}$ where $\varepsilon^{2}=0.01$. Then for every pair $(r, s)$ we started with the initial data $u(\tilde{x}, 0)=v(\tilde{x}, 0)=0.05 \cos \left(k_{c} x\right)$ and studied the evolution in time. We find that for $0.75 \leq s \leq 0.9$ the initial function is stable, where the amplitude of $u$ decreases to 0.035 (and for $v$ it still is 0.05 ); see figure $7 \mathrm{~b}$ for the $v$-component of the stable, periodic solutions for $s=0.82$.

For $s>0.9$, we find, because $s$ is coming closer to $s_{c G L}$ that, the solutions are influenced by the dynamics of the coupled GL-system (3.25) which governs the behaviour for $s$ close to $s_{c G L}(\sim 0.94)$. We see here that the amplitude of the stable solution is periodic (see figure $7 \mathrm{a}$ ). For $0.71 \leq s \leq 0.75$, the number of maxima of the stable solution is the same as the number of maxima of the initial periodic solution. However, the stable stationary solutions are no longer periodic; they have a 'multi-bump' structure. In figure $7 \mathrm{c}$ the $v$-component of the stable solution is given for $s=0.72$.

Thus for $s$ not too close to $s_{E F K}$ or to $s_{C G L}$, the numerical results coincide with the theoretical results which are known for the GL-equation. And for $s$ close to $s_{e F K}$ or $s_{C G L}$ we find that the eFK2-equation resp. the coupled system of GL-equations influences the behaviour of the stable solution considerably.

The predictions of the GL-theory can also be checked by fixing $s$ (and thus $r$ ) and changing the period of the initial data. Theoretically, we know that around $k=k_{c}$ there is a whole interval of $k$-values for which there are stable stationary periodic solutions $\cos (k x)$, see Appendix B. We fixed $s=0.8 ; r=-1.24$ and started with an initial periodic cosine-function which has a integer number of periods in the interval. We changed this number from 8 to 48 periods. From the simulations we see that periodic solutions which start with a number of periods that is between 21 and 31 (including 21 and 31 ) are stable. Initial data which have more or less periods in the interval are not stable and will go to solutions which have a number of periods in between 21 and 31 .

These results more or less coincide with the theoretical results. However, theoretically the interval of number of periods where the solution is stable is slightly different. The number of periods has to lie (theoretically) between 21.4 and 33.5 periods, see Appendix B. The observation that the interval of stable solutions differs from what could be expected by the theoretical 
predictions for the GL-equation might be explained by the influence of the singularly perturbed GL-equation. The interval of numerically stable solutions is shifted to the left with respect to the interval of theoretically stable solutions of the GL-equation. This is exactly what is shown for the singularly perturbed GL-equation, see Appendix B. We will not study this in detail because for $s=0.8$ the singularly perturbed GL-equation can only be expected to have a very small influence on the solutions.

\subsection{Numerical simulations near $\left(r_{e F K}, s_{e F K}\right)$}

So far, we showed numerical simulations for $s$ in the region where we derived the GL-equation. However, we already observed some 'strange' behaviour for $s \leq 0.74$ when starting with initial data $u(\tilde{x}, 0)=v(\tilde{x}, 0)=0.05 \cos \left(k_{c} x\right)$. This is ascribed to the influence of the eFK2-equation. Now, we will study this influence further. We will start with fixed initial data and vary the pair $(r, s)$ from $(-0.96 ; 0.94)$ to $(-1.48 ; 0.68)$ where we decrease $s$ with steps of 0.1 (or sometimes larger steps) and take $r=r_{c}(s)-\varepsilon^{2}$. We performed these simulations for initial data with 8,10 and 12 periods. We found that when taking $0.77 \leq s \leq 0.94$, the initial data evolve towards a stable, sinusoidal periodic solution; for $0.69 \leq s \leq 0.76$ the simulations exhibit a non-periodic stable solution, for $s$ even smaller we find a constant solution. The transition from $s=0.77$ to $s=0.76$ is quite drastic, see figures $8 \mathrm{a}$ and b (and section $5.4)$.

First we focus on $0.77 \leq s \leq 0.94$. Here we observe stable periodic solutions, however, the number of periods of the stable solution is not the same as in the initial data. We also see that the number of periods at the end of the simulation depends on the number of periods of the initial function. Theoretically, we would expect that the end-period lies in an interval which is symmetric around $\frac{2 \pi}{k_{c}}$, this is the period of the stable solution $\cos \left(k_{c} x\right)$ where $k_{c}$ depends on $s$. We find that, except for $s=0.82$, the end-periods lie in the theoretical stable interval. For $s=0.82$, the periodic stable solution must have, according to theory, from 26.8 to 33.7 periods. We find a stable function with 25 periods if we take an initial condition with 8 and 12 periods (taking an initial condition with 10 periods, we find a stable function with 30 periods, this is in the interval of theoretically stable solutions)-see also section 5.4. This could be ascribed to the same phenomenon which shifted the theoretical interval of stable solutions which we found before for $s=0.8$.

Now, we will look at the behaviour of the solutions for $0.69 \leq s \leq 0.76$. The number of maxima of the stable solution remains the same as in the initial data, see figures 8, 9 and 10. Again we see that the stable function depends on the number of periods of the initial function. Some of the simulations result in a periodic stable solution. However, these solutions are 
not a 'simple' sin- or cosine-like function as in the GL case, but so-called multi-bump solutions. When starting with 10 periods where $s=0.69$ (figure $9 \mathrm{~b}$ ) and with 12 periods where $s=0.69$ and $s=0.7$, the periodic solutions have maxima which are sharp peaks and minima which are much smoother. For initial data with 8 periods where $s=0.71$ and $s=0.72$ (figure $8 \mathrm{c}$ ) and with 10 periods where $s=0.74, s=0.75$ and $s=0.76$ (figure 9a) the solutions still have sharp peaks as maxima. At the minima we see that a 'dip' appears. For the other initial data, the stable solutions are not periodic. However, we see repeating patterns similar to the ones we saw before. The maxima are always sharp peaks and the minima are either smoother or have $\mathrm{a}$ 'dip' (figures 8b and $\mathrm{d}$ and 10a and $\mathrm{b}$ and section 5.4). All the stable solutions are symmetric in the middle of the $x$-interval.

\subsection{Numerical simulations with cubic nonlinearities}

In section 3.4 we derived an eFK-equation with cubic non-linear terms, this followed by setting $\alpha_{1}, \cdots, \beta_{3}$ equal to zero in the non-linear terms $N_{1}$ and $N_{2}((3.1)$ and $(3.2))$. Because the eFK3-equation is studied extensively in literature $([24,8,18,7])$, we also did some numerical simulations in this case, where the eFK3-equation is expected to describe the behaviour of small solutions. We started with an initial function with 6 periods and decreased $s$ from $s=0.77$ to $s=0.67$ where we take steps of size 0.01 . Except for $s=0.68$ and $s=0.67$, the stable solutions are periodic, but, these solutions are not all 'simple' sin- or cosine-like functions, once again multi-bump solutions occur. For $s=0.77$ and $s=0.76$ we do find a 'simple' cosine-like function. In the transition to $s=0.75$ the stable solution changes drastically, see figures $11 \mathrm{a}$ and $\mathrm{b}$. For $0.71 \leq s \leq 0.75$ the solutions have both at the maxima and at the minima a 'dip', see figure $11 \mathrm{~b}$.

For $s=0.7$ and $s=0.69$, this dip vanishes at the maxima and still remains at the minima (see figure 11c). Note that this solution is not symmetric in the $x$-axis.

We also performed numerical simulations for other initial data. Similar behaviour as described above is found. We fixed $s=0.75$ changing the period of the initial condition gives interesting stable solutions when starting with 4 and 8 periods, see figures $1 \mathrm{a}$ and $\mathrm{b}$. However, when starting with 12 or more periods, nearly all the exotic behaviour has vanished and the stable solutions become periodic cosine-functions.

\subsection{Interpretation}

At the beginning of section 5 we remarked that the outcome of the simulations did not really depend on the choices of $N_{1}(u, v)$ and $N_{2}(u, v)$. We for instance chose $\alpha_{1}, \ldots, \beta_{3}, a, b$ such that the sign of the quadratic term in 
the eFK2-equation changed (with respect to the standard choice of figures $8,9,10)$. This yields the expected outcome: we found that the observed multi-bump patterns are in essence the same, except for the fact that the $v$ components of the stable patterns are now reflected in the $x$-axis. However, in choosing $N_{1}(u, v)$ and $N_{2}(u, v)$ it is crucial that $\sqrt{d} \alpha-\beta$ (see (3.21) and (4.4)) is small. If that is not the case then the simulations follow the predictions of the asymptotic theory (see section 4.1): the Landau coefficient does not change sign near $\left(r_{e F K}, s_{e F K}\right)$, thus, there are no bounded small solutions between $\left(r_{n l}, s_{n l}\right)$ and $\left(r_{e F K}, s_{e F K}\right)$ for $r$ near and below $r_{c}(s)$.

A priori, one might guess that the fact that $\sqrt{d} \alpha=\beta$ is small will not influence the asymptotic analysis too much. However if $\sqrt{d} \alpha-\beta$ is 'numerically' small, then it is not clear whether one can neglect the asymptotic higher order terms of the eFK2-equation $(3.21)$ of $O(\delta)$. On the contrary, it can be expected that at least some of these terms cannot be neglected. Of course, this problem can be circumvented by only considering cubic nonlinearities $N_{1}$ and $N_{2}$, then $\sqrt{d} \alpha-\beta \equiv 0$ (see (3.10) and (3.11)). Here the above problem does not occur: the asymptotic dynamics are described by the eFK3-equation (see section 3.4). In figure 12a we show a plot of the outcome of a numerical simulation of the eFK3-equation. Here the coefficients and initial condition are comparable to the choices we made when performing the numerical simulations on (2.1) that produced the solution given in figure 1a. We know by section 3.4 that the behaviour of these solutions should be described asymptotically by the eFK3-equation. There is a striking resemblance between figures $12 \mathrm{a}$ and $1 \mathrm{a}$ (at least qualitatively). This strongly suggests that the attractors of the eFK3-equation should also be 'approximations' of the attractors of the full system (1.4) near $\left(r_{e F K}, s_{e F K}\right)$ (for cubic $N_{1}$ and $N_{2}$ ). Of course, we are still far away from a mathematical proof of such a statement.

A full asymptotic analysis of the more 'generic' case of quadratic nonlinearities is the subject of future research. The analysis will become much more involved: $\sqrt{d} \alpha-\beta$ should be considered as a third small quantity. This yields that the 'non-linear' degenerate GL bifurcation described in section $4.1([16,9,27])$ occurs asymptotically close to the eFK bifurcation point. Thus, the analysis of this paper should be combined with the approach of $[16,9]$. As a result, one expects that the dynamics near $\left(r_{e F K}, s_{e F K}\right)$ are described by a combination of the eFK2, the eFK3 and the degenerate GLequation (4.5). This is also supported by a numerical simulation of the 'eFK2+3' equation:

$$
B_{t}=B+D B_{x x}-B_{x x x x}+k B^{2}+l B^{3}
$$

We again consider the situation (and initial conditions) similar to that described by the asymptotic eFK2-equations of this section. Then, we find 
that there are no bounded solutions for $l=0$ (and $D<0$ ). Note that this agrees with the above: this is the case that $\sqrt{d} \alpha-\beta$ is not small. Thus, the existence of a (negative, possibly small) cubic term is of crucial importance. In figure $12 \mathrm{~b}$ we show an attractor of (5.1) for $k=0.5, l=-1$ and $D=-2$. At this point it is not clear whether this is a pattern that can also be found in the simulations of (3.21): it is certainly not exactly like those shown in figures $8,9,10$. A full analysis will yield all relevant non-linear terms of the 'degenerate' eFK-equation (one might, for instance, also expect the appearance of 'Kuramoto-Sivashinsky terms' as $\left.\left(B_{x}\right)^{2}\right)$.

Another interesting phenomenon that we so far did not discuss is the sharp transition between the 'regular' GL patterns and the multi-bump patterns: compare for instance figure $8 \mathrm{a}$ to $8 \mathrm{~b}$, here the only difference is a 0.02 change in $s$ (or figure 11a to figure 11b: $\Delta s=0.01$ ). This transition is - at least numerically - closely related to the process by which an unstable periodic pattern of the (real) GL-equation evolves towards a stable periodic pattern. This process has been described by Kramer and Zimmerman in [19], but that description does not seem to be accurate enough to understand it completely - at least not in the context of this paper (note that the transition occurs in a region where the GL-equation should be replaced by the singularly perturbed GL-equation (4.3)). Initially there is no significant difference in the numerical simulations leading to figures $8 \mathrm{a}$ and $8 \mathrm{~b}$ : both unstable cosines of the initial conditions form 'dips' either at the maxima or the minima, and start to look like a multi-bump pattern. However, in the simulation leading to figure 8 a these 'dips' grow until a sinusoidal pattern appears. In the simulation leading to figure $8 \mathrm{~b}$ the 'dips' stop to grow at a certain level and the stable multi-bump pattern is formed. The same mechanism seems to be responsible for all multi-bump patterns observed in this paper. Thus, a more detailed understanding of the process by which the GL-equation brings a periodic pattern from outside the Eckhaus band into this band of stable solutions would shed more light on the creation of stable multi-bump solutions (and vice versa).

Finally, we remark that changing the length of the $x$-interval has a very subtle influence on the numerical simulations of this process and thus on the type of the observed asymptotically stable multi-bump patterns: the 'dips' appear 'suddenly' at different places, resulting either in an periodic GL pattern with an unexpected number of periods, or a structurally different multi-bump pattern.

Acknowledgement: The authors thank Paul Zegeling, for teaching them how to use the 'moving-grid' code described in [1] and for performing the simulations on the eFK-equations (figure 12). 


\section{A Appendix}

Here we give a detailed derivation of the GL-equation. First we express the functions in expansion (3.5) in terms of $A$ by solving the equations at the $a_{1}=2$-level. For $a_{1}=2, b_{1}=0$, we get

$$
\left\{\begin{aligned}
r_{c} X_{02}+Y_{02}+2 \alpha|A|^{2} & =0 \\
s Y_{02}-X_{02}+2 \beta|A|^{2} & =0
\end{aligned}\right.
$$

where

$$
\begin{aligned}
& \alpha=d \alpha_{1}+\sqrt{d} \alpha_{2}+\alpha_{3} \\
& \beta=d \beta_{1}+\sqrt{d} \beta_{2}+\beta_{3} .
\end{aligned}
$$

From this system we can solve $X_{02}$ and $Y_{02}$ :

$$
\left(\begin{array}{c}
X_{02} \\
Y_{02}
\end{array}\right)=-\frac{2 d|A|^{2}}{(s-\sqrt{d})^{2}}\left(\begin{array}{c}
s \alpha-\beta \\
\alpha+\beta r_{c}
\end{array}\right) .
$$

On the $a_{1}=b_{1}=2$-level we have:

$$
\left\{\begin{array}{l}
r_{c} X_{22}+Y_{22}-4 k_{c}^{2} X_{22}+\alpha A^{2}=0 \\
s Y_{22}-X_{22}-4 k_{c}^{2} d Y_{22}+\beta A^{2}=0 .
\end{array}\right.
$$

Solving $X_{22}$ and $Y_{22}$ from this system:

$$
\left(\begin{array}{c}
X_{22} \\
Y_{22}
\end{array}\right)=\frac{d A^{2}}{9(s-\sqrt{d})^{2}}\left(\begin{array}{c}
(3 s-4 \sqrt{d}) \alpha+\beta \\
-\alpha+\frac{\beta}{d}(3 s-2 \sqrt{d})
\end{array}\right) .
$$

For $a_{1}=2, b_{1}=1$ we find

$$
\mathcal{M}_{c}\left(\begin{array}{c}
X_{12} \\
Y_{12}
\end{array}\right)=-2 i k_{c} A_{\xi_{A}}\left(\begin{array}{c}
\sqrt{d} \\
1
\end{array}\right) .
$$

This equation automatically satisfies the solvability condition (3.4):

$$
\left(\begin{array}{c}
X_{12} \\
Y_{12}
\end{array}\right)=\left(\begin{array}{c}
2 i d k_{c} A_{\xi_{A}} \\
0
\end{array}\right)+\left(\begin{array}{c}
\sqrt{d} \\
1
\end{array}\right) A_{2}\left(\xi_{A}, \tau\right)
$$

Where $A_{2}$ is a second higher order amplitude which depends on $\xi_{A}$ and $\tau$. Finally, on the $a_{1}=3, b_{1}=1$-level, the modulation equation is derived from

$$
\begin{aligned}
& \mathcal{M}_{c}\left(\begin{array}{c}
X_{13} \\
Y_{13}
\end{array}\right)=\left(\begin{array}{c}
\sqrt{d} \\
1
\end{array}\right) A_{\tau}+\left(\begin{array}{c}
v_{1} \sqrt{d} \\
0
\end{array}\right) A-\left(\begin{array}{c}
\sqrt{d} \\
d
\end{array}\right) A_{\xi_{A} \xi_{A}}-2 i k_{c}\left(\begin{array}{c}
X_{12 \xi_{A}} \\
d Y_{12 \xi_{A}}
\end{array}\right) \\
& -\left(\begin{array}{c}
2 \alpha_{1} \sqrt{d}\left(x_{02}+x_{22}\right)+\alpha_{2}\left(\sqrt{d}\left(y_{02}+y_{22}\right)+x_{02}+x_{22}\right)+2 \alpha_{3}\left(y_{02}+y_{22}\right) \\
2 \beta_{1} \sqrt{d}\left(x_{02}+x_{22}\right)+\beta_{2}\left(\sqrt{d}\left(y_{02}+y_{22}\right)+x_{02}+x_{22}\right)+2 \beta_{3}\left(y_{02}+y_{22}\right)
\end{array}\right) A|A|^{2} \\
& +3\left(\begin{array}{c}
a d \sqrt{d} \\
b
\end{array}\right) A|A|^{2},
\end{aligned}
$$


where $\left(\begin{array}{c}X_{02} \\ Y_{02}\end{array}\right)=|A|^{2}\left(\begin{array}{c}x_{02} \\ y_{02}\end{array}\right)$ and $\left(\begin{array}{c}X_{22} \\ Y_{22}\end{array}\right)=A^{2}\left(\begin{array}{c}x_{22} \\ y_{22}\end{array}\right)$. Setting $F=$ $x_{02}+x_{22}$ and $G=y_{02}+y_{22}$ and applying the solvability condition (3.4) leads to

$$
\begin{array}{r}
(d-1) A_{\tau}+v_{1} \sqrt{d} A-2 i k_{c}\left(\sqrt{d} X_{12 \xi_{A}}-d Y_{12 \xi_{A}}\right)+\left(\left(2 \beta_{1} \sqrt{d}+\beta_{2}-2 \alpha_{1} d-\sqrt{d} \alpha_{2}\right) F\right. \\
\left.+\left(\sqrt{d} \beta_{2}+2 \beta_{3}-d \alpha_{2}-2 \sqrt{d} \alpha_{3}\right) G+3\left(a d^{2}-b\right)\right) A|A|^{2}=0 .
\end{array}
$$

which yields equations (3.6)-(3.11).

\section{B Appendix}

Here we briefly describe results on the stability of periodic solutions of the GL-equation. It is known that for a general GL-equation

$$
A_{\tau}=R A+b A_{\xi \xi}+l A|A|^{2}
$$

there exist periodic solutions of the form

$$
A= \pm \rho e^{i k \xi} \text { where } \rho^{2}=\frac{b k^{2}-R}{l} .
$$

These periodic solutions are stable as long as $k^{2}<\frac{R}{3 b}$ (see [12]). This gives us the stable periodic solutions of the GL-equation (3.6) where $R, b$ and $l$ can be expressed in terms of $d, s$ and $h$ (we set $v_{1}=1$ ):

$$
R=\frac{\sqrt{d}}{1-d}, b=\frac{4 \sqrt{d}(s-\sqrt{d})}{1-d}, l=\frac{h}{1-d} .
$$

Thus, for $k^{2}<\frac{1}{12(s-\sqrt{d})}$ the periodic solutions $A= \pm \rho e^{i k \xi}$ with $\rho^{2}=$ $\frac{4 \sqrt{d}(s-\sqrt{d}) k^{2}-\sqrt{d}}{h}$ of the GL-equation (3.6) are stable. Now we want to find stable periodic solutions of the reaction-diffusion system (2.1). Therefore we use the expansion

$$
\left(\begin{array}{l}
u \\
v
\end{array}\right)=\varepsilon\left(\begin{array}{c}
\sqrt{d} \\
1
\end{array}\right)\left(A e^{i k_{c} x}+\bar{A} e^{-i k_{c} x}\right)+h o t
$$

where $k_{c}^{2}=\frac{s-\sqrt{d}}{d}$ (see section 3.1). Substituting the expression which we found for $A$, gives for $v$ (observe that $u=\sqrt{d} v$ at leading order)

$$
v=2 \varepsilon \rho \cos \left(\left(k \varepsilon+k_{c}\right) x\right)
$$

where we used the fact that $\xi=\varepsilon x$. This solution is stable as long as $k^{2}<\frac{1}{12(s-\sqrt{d})}$. Thus, fixing $d$ and $s$ (which are the only parameters left in this problem), the family of stable periodic solutions can be calculated. 
For example, setting $d=0.5$ and $s=0.8$, as we do in the simulations in section 5.1, we obtain $k_{c}=0.431$ and stability for $k^{2}<0.897$. Thus the solutions $a \cos (m x)$ are stable when $0.336 \leq m \leq 0.526$ where $a=2 \varepsilon \rho$ (here we used the fact that in the numerical simulations $\varepsilon=0.1$ ). This coincides with a cosine-function which has from 21.4 to 33.5 periods in the interval $[0,400]$.

For the singularly perturbed GL-equation stability of periodic solutions can also be given. We will only state the results here, the calculations go along the same line as for the classical GL-equation. We can rescale the singularly perturbed GL-equation (4.3), by rescaling $\tau, \xi$ and $A$, to the following equation

$$
A_{\tau}=A+A_{\xi \xi}-2 i b A_{\xi \xi \xi}-b^{2} A_{\xi \xi \xi \xi}-l A|A|^{2}
$$

where $l= \pm 1$ and $0<b \ll 1$. Then there exist periodic solutions of the form $A=R e^{i k \xi}$ where $R^{2}=1-k^{2}(b k+1)^{2}$. These solutions are stable for $-\frac{1}{9} b-\frac{1}{\sqrt{3}}<k<-\frac{1}{9} b+\frac{1}{\sqrt{3}}$ (for the non-singular GL-equation the solutions are stable for $k^{2}<\frac{1}{3}$ ). Thus, the interval of $k$-values for which the solution $R e^{i k \xi}$ is stable has shifted to the left.

\section{References}

[1] J.G. Blom, P.A. Zegeling (1994) Algorithm 731: A moving-grid interface for systems of one-dimensional time-dependent partial differential equations, ACM Transactions in Mathematical Software 20, 194-214.

[2] E. Bodenschatz, W. Zimmerman, L. Kramer (1988) On electrically driven pattern-forming instabilities in planar nematics, J. Phys. (France) 49,1875-1899.

[3] P. Bollerman, A. van Harten and G. Schneider (1994) On the justification of the Ginzburg-Landau approximation, in Nonlinear Dynamics and Pattern Formation in the Natural Environment (A. Doelman and A. van Harten eds.), Pitman Res. Notes in Math. 335, UK, 20-36.

[4] B. Buffoni, A.R. Champneys, J.F. Toland (1996) Bifurcation and coalescence of a plethora of homoclinic orbits for a hamiltonian system, $J$. of Dyn. Diff.Eq. 8 221-279.

[5] P. Collet, J.P. Eckman (1990) The time-dependent amplitude equation for the Swift-Hohenberg problem, Comm. Math. Phys. 132, 139-153.

[6] E.D. Conway (1984) Diffusion and predator-prey interaction: patterns in closed systems, in Partial differential equations and dynamical sys- 
tems (W.E. Fitzgibbon ed.),Pitman Res. Notes in Math. 101, UK, 85133.

[7] P. Coullet, C. Elphick, D. Repaux (1987) Nature of spatial chaos Phys. Rev. Lett. 58, 431-434.

[8] G.T. Dee, W. van Saarloos (1988) Bistable systems with propagating fronts leading to pattern formation, Phys. Rev. Lett. 60, 2641-2644.

[9] A. Doelman, W. Eckhaus (1991) Periodic and quasi-periodic solutions of degenerate modulation equations, Physica D 53, 249-266.

[10] A. Doelman, T.J. Kaper, P.A. Zegeling (1997) Pattern formation in the one-dimensional Gray-Scott model, Nonlinearity 10, 523-563.

[11] A. Doelman, V. Rottschäfer (1996) Singularly perturbed and non-local modulation equations for system with interacting instability mechanisms, to appear in J. Nonlinear Sci.

[12] W. Eckhaus (1965) Studies in non-linear stability theory, Springer Verlag, New York.

[13] W. Eckhaus (1979) Asymptotic analysis of singular perturbations, North-Holland, Amsterdam.

[14] W. Eckhaus (1992) On modulation equations of the Ginzburg-Landau type, in ICIAM 91: Proc. 2nd Int. Conf. Ind. Appl. Math. (R.E. O’Malley ed.), 83-98.

[15] W. Eckhaus (1993) The Ginzburg-Landau manifold is an attractor, $J$. Nonlinear Sci. 3 329-348.

[16] W. Eckhaus, G. Iooss (1989) Strong selection or rejection of spatially periodic patterns in degenerate bifurcations, Physica D 39, 124-146.

[17] A. van Harten (1991) On the validity of the Ginzburg-Landau's equation J. Nonlinear Sci. 1, 397-422.

[18] W.D. Kalies, R.C.A.M. VanderVorst (1996) Multitransition homoclinic and heteroclinic solutions of the extended Fisher-Kolmogorov equation, J.Diff.Eq. 131, 209-228.

[19] L. Kramer, W. Zimmerman (1985) On the Eckhaus instability for spatial periodic patterns, Physica D 16, 221-232.

[20] Y. Kuramoto (1984) Chemical oscillations, Waves and Turbulence, Springer Verlag, New York.

[21] J. Lega, J.V. Moloney, A.C. Newell (1994) Swift-Hohenberg equation for lasers, Phys.Rev.Let. 73, 2978-2981. 
[22] J. Lega, J.V. Moloney, A.C. Newell (1995) Universal description of laser dynamics near threshold, Physica D 83, 478-498.

[23] A.C. Newell, J.A. Whitehead (1969) Finite bandwidth, finite amplitude convection, J. Fluid Mech. 28, 279-303.

[24] L.A. Peletier, W.C. Troy (1995) Spatial patterns described by the extended Fisher-Kolmogorov equation: Periodic solutions, to appear in SIAM J.Math.Anal..

[25] W. van Saarloos, P.C. Hohenberg (1992) Fronts, pulses, sources and sinks in generalised complex Ginzburg-Landau equations, Physica $D$ 56, 303-367.

[26] G. Schneider (1996) Hopf-bifurcation in spatially extended reactiondiffusion systems, to appear in J. Nonlinear Sci.

[27] A. Shepeleva (1996) On the validity of the degenerate Ginzburg-Landau equation, to appear in Math. Meth. Appl. Sc.

[28] J. Swift, P.C. Hohenberg (1977) Hydrodynamic fluctuations at the convective instability, Phys.Rev.A 15, 319-328.

[29] A.M. Turing (1952) The chemical basis of morphogenesis, Phil. Trans. Roy. Soc. Lond., Series B, 237, 37-72.

[30] W. Zimmerman (1991) Propagating fronts near a Lifshitz point, Phys. Rev. Lett. 66, 1546.

[31] W. Zimmerman, D. Armbruster, L. Kramer, W. Kuang (1988) The effect of spatial modulations on codimension-2 bifurcations, Europhys. Lett. 6, 505-510. 

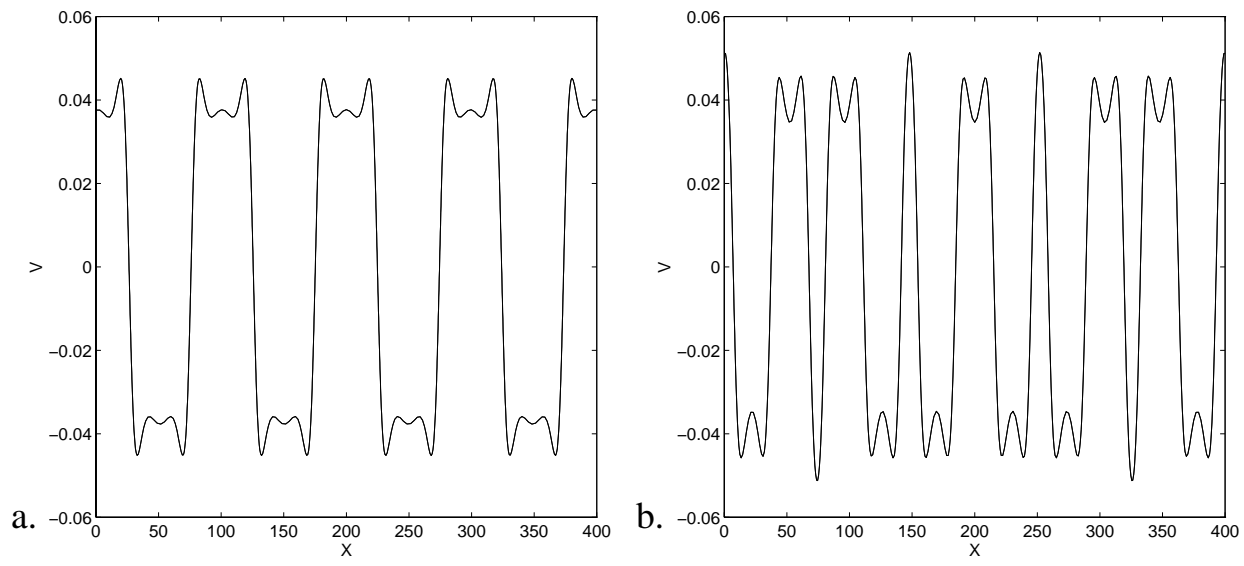

Figure 1: The $v$-component of the asymptotically stable (small) solutions to (1.4) for $(r, s)$ close to the co-dimension 2 point $(s=0.75)$. In section 3.4 it will be shown that these solutions are described by the eFK3-equation. The only difference between $\mathrm{a}$. and $\mathrm{b}$. is the choice of initial conditions: a. 4 periods b. 8 periods (we refer to the caption of figure 11 for more details).

a.

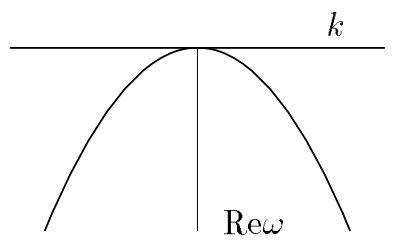

b.

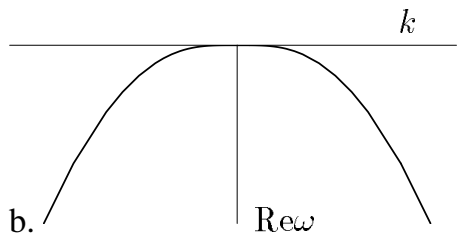

c.

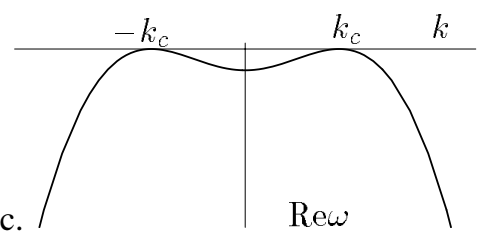

d.

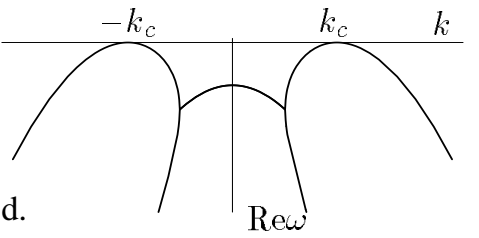

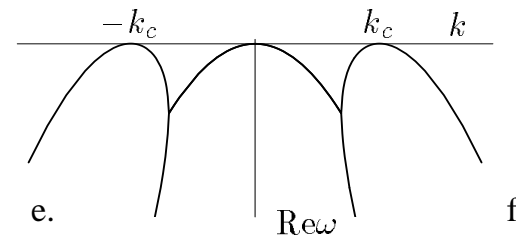

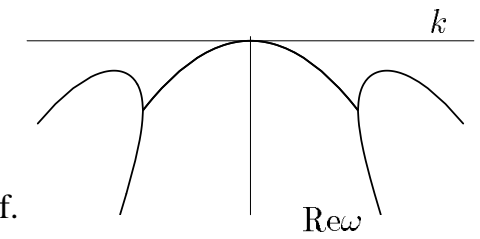

Figure 2: The real part of the eigenvalue-curves given as a function of $k$ for different choices of the pair $(r, s)$. a. On $\Gamma_{1}$ where $s$ is not close to $s_{e F K}$ b. $s=s_{e F K}$ c. On $\Gamma_{2}, s_{e F K}<s<\tilde{s}$ d. On $\Gamma_{2}, \tilde{s}<s<s_{c G L}$ and not close to $s_{c G L}$ e. For $s=s_{c G L}$ f. On $\Gamma_{3}$. Note that only $\omega_{1}$ has been plotted in a-c. 


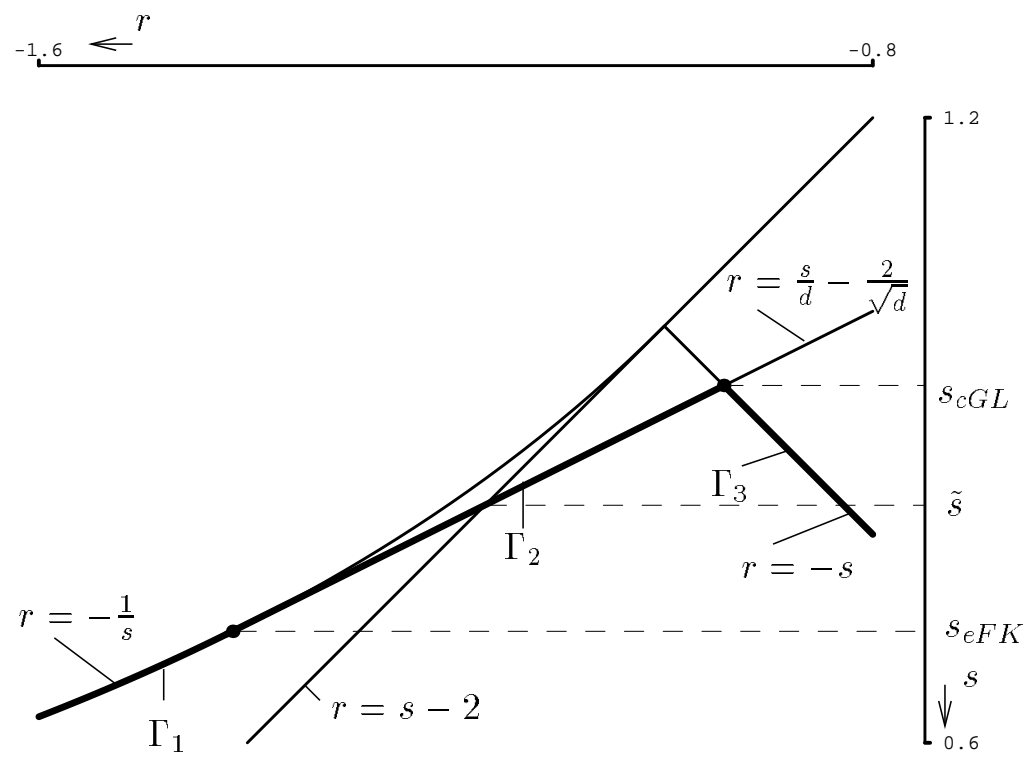

Figure 3: The $(r, s)$-plane for $d<1$. The bifurcation curves $\Gamma_{1}, \Gamma_{2}$ and $\Gamma_{3}$ are the thicker lines. 


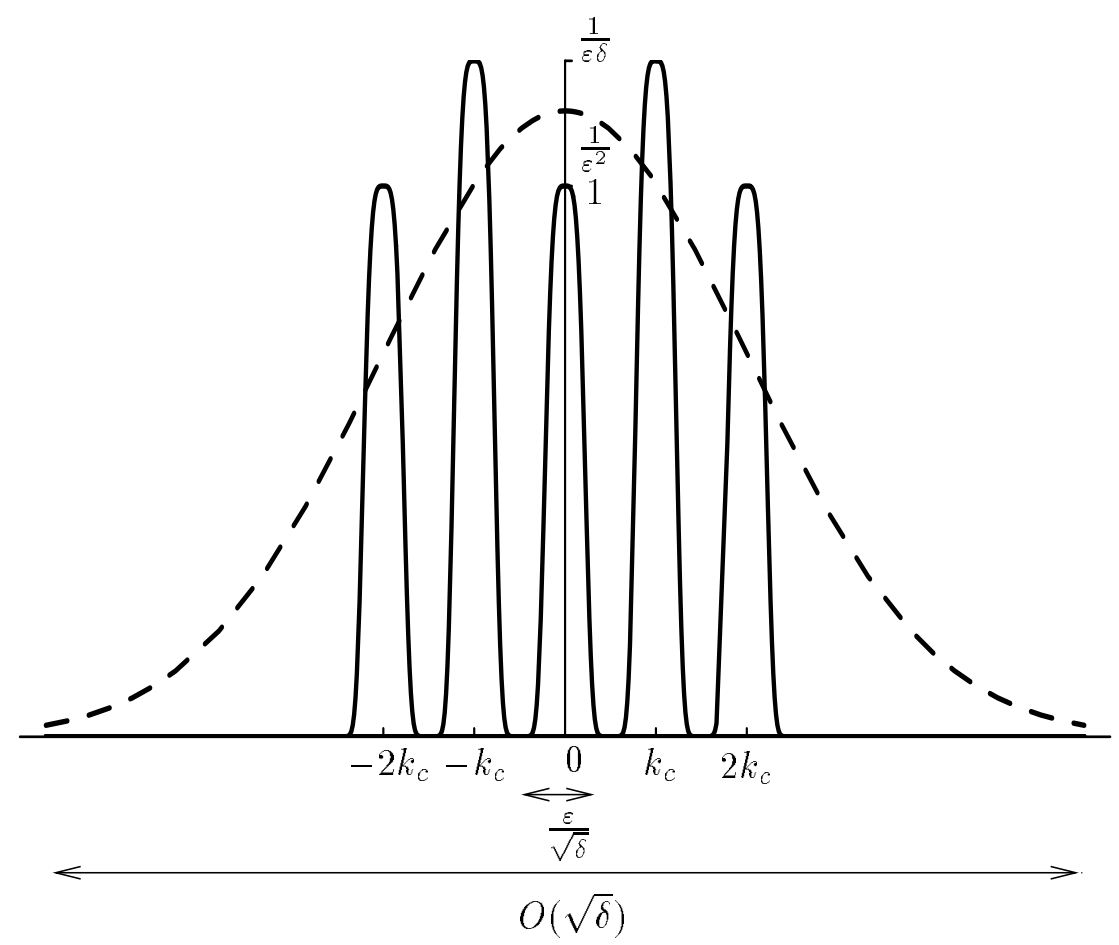

Figure 4: The GL-decomposition (the peaks) and the eFK-decomposition (the dotted curve) given in Fourier space. In the GL-decomposition the peaks around $k=k_{c}$ are of height $\frac{1}{\varepsilon \delta}$ and around $k=0, \pm 2 k_{c}$ of height 1 . All these peaks are of width $O\left(\frac{\varepsilon}{\sqrt{\delta}}\right)$. The eFK-decomposition is one "wide' peak of height $\frac{1}{\delta^{2}}$ and width $\sqrt{\delta}$. 


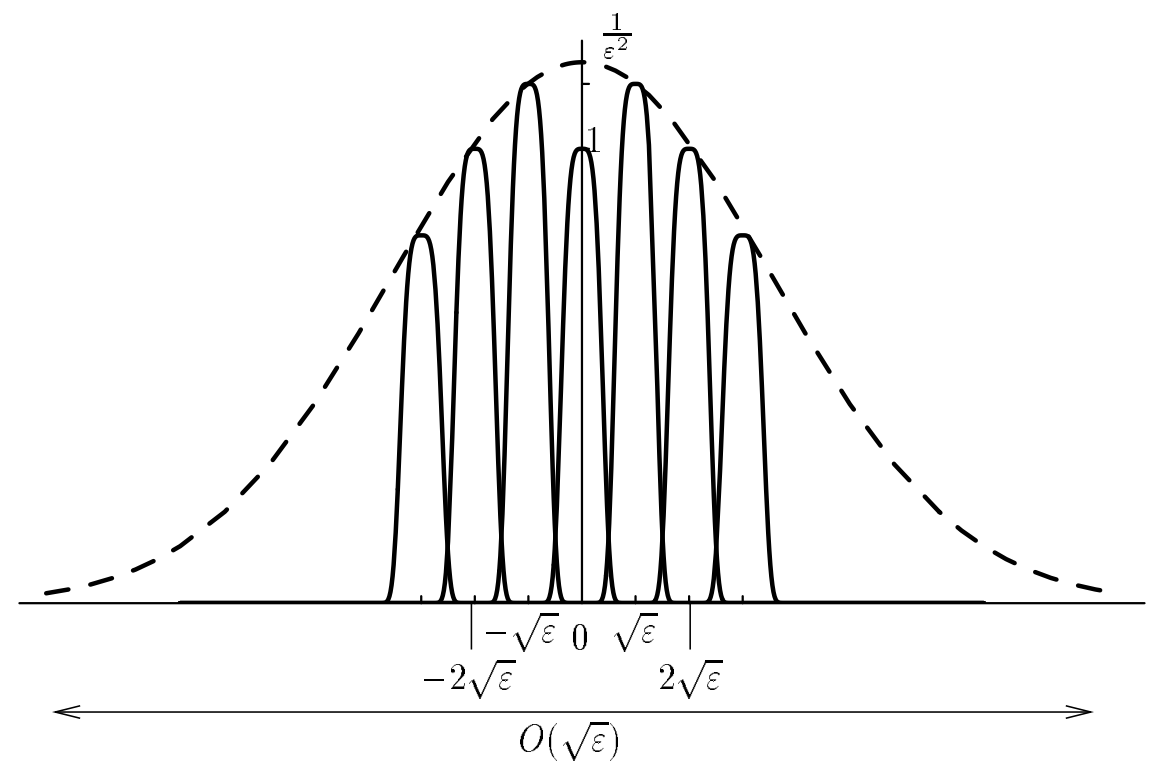

Figure 5: The GL-decomposition (the peaks) and the eFK-decomposition (the dotted curve) given in Fourier space in the limit $\delta \downarrow O(\varepsilon)$. The peaks of the GL-decomposition start to overlap and become of height $\frac{1}{\varepsilon^{2}}$, which is the same as the height of the 'wide' curve of the eFK-decomposition. All the peaks of the GL-decomposition together form the 'wide' curve of the eFK-decomposition. 

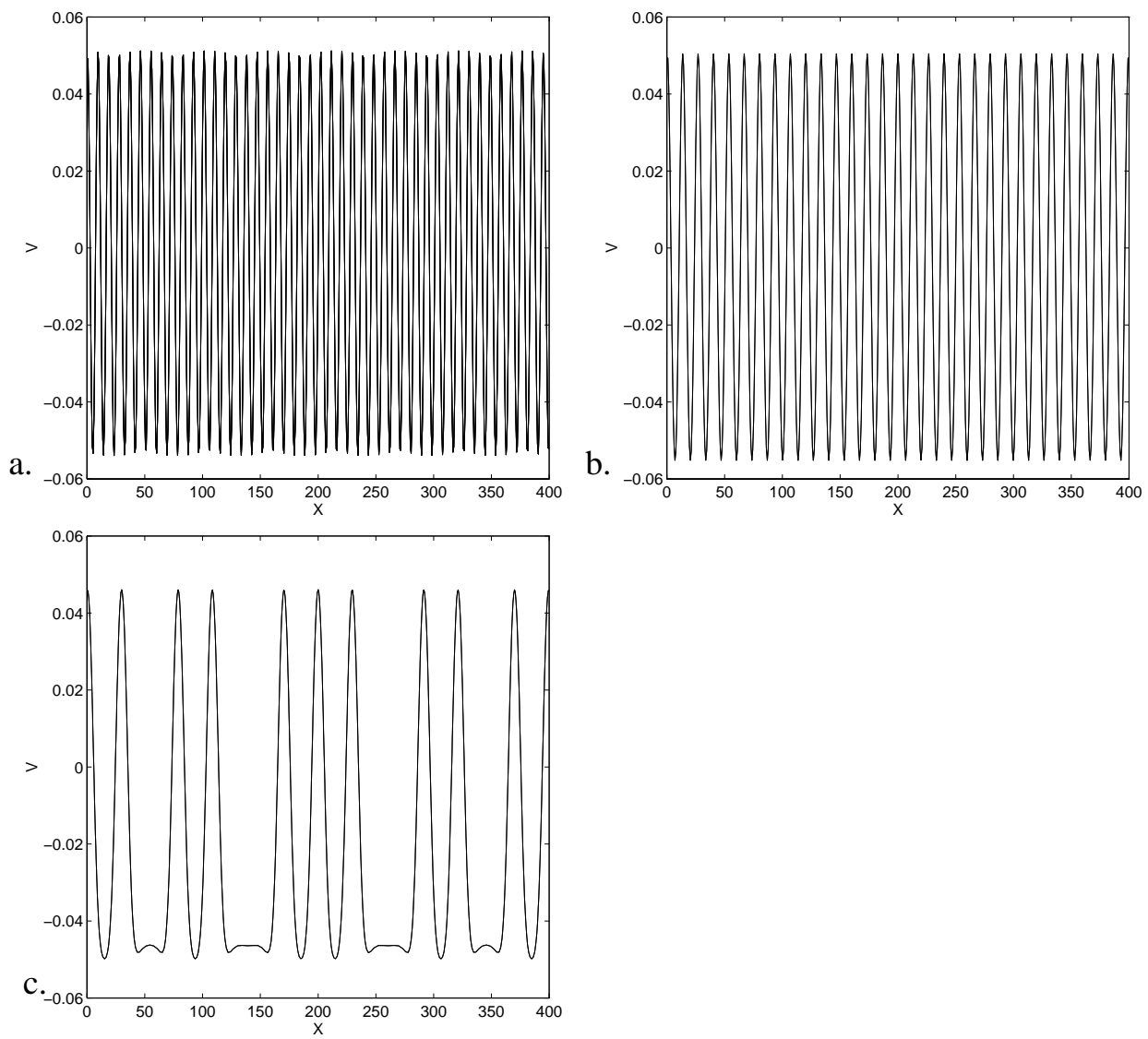

Figure 6: The $v$-component of the stable solutions when starting with the initial data $0.05 \cos \left(k_{c} x\right)$ for a. $\mathrm{s}=0.94$ b. $\mathrm{s}=0.82 \mathrm{c}$. $\mathrm{s}=0.72$. Here $\alpha_{1}=\alpha_{3}=$ $\beta_{1}=\beta_{3}=0, \alpha_{2}=0.71, \beta_{2}=0.5, a=1$ and $b=3$. 

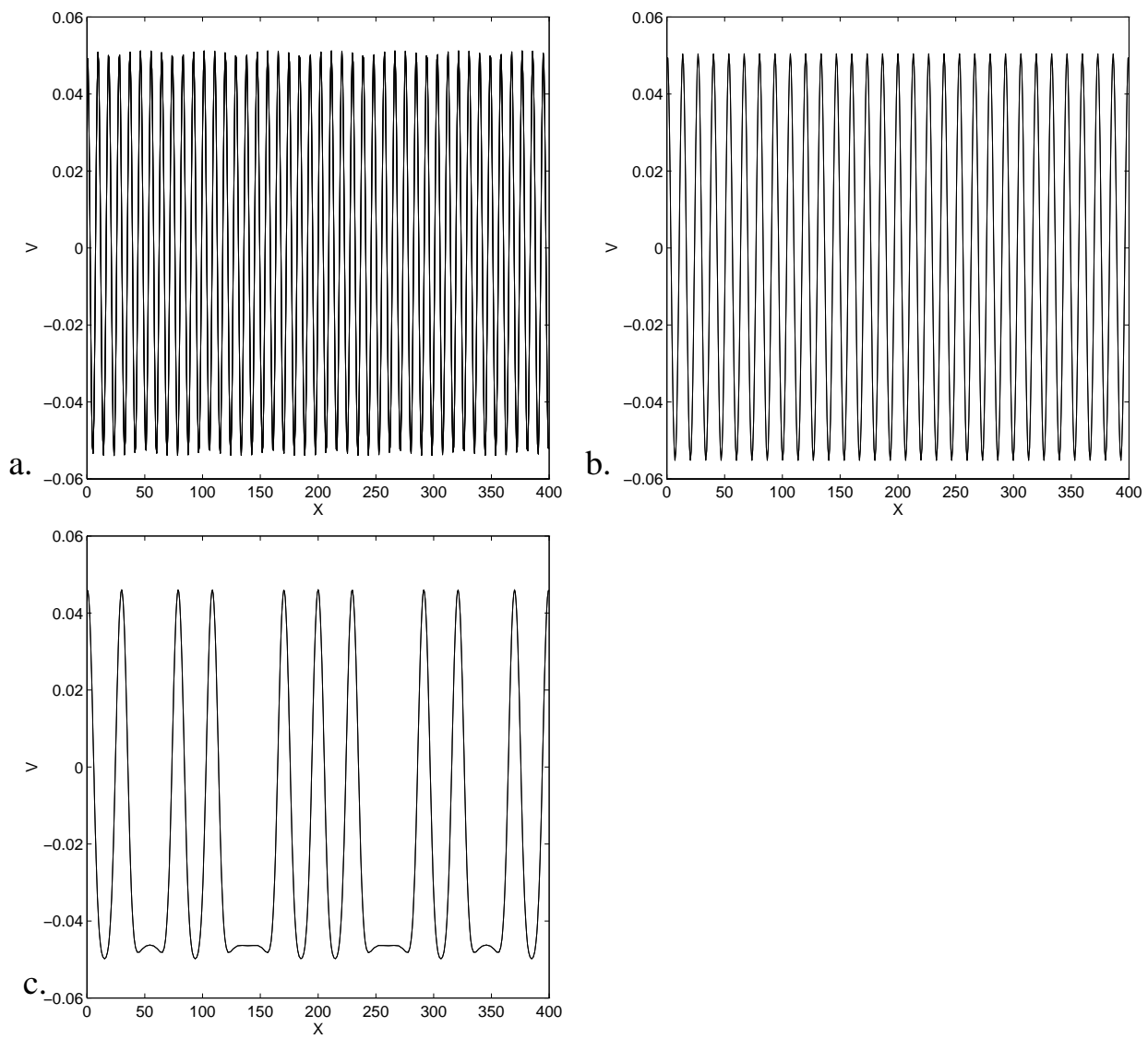

Figure 7: The $v$-component of the stable solutions when starting with the initial data $0.05 \cos \left(k_{c} x\right)$ for a. $\mathrm{s}=0.94$ b. $\mathrm{s}=0.82 \mathrm{c}$. $\mathrm{s}=0.72$. Here $\alpha_{1}=\alpha_{3}=$ $\beta_{1}=\beta_{3}=0, \alpha_{2}=0.71, \beta_{2}=0.5, a=1$ and $b=3$. 

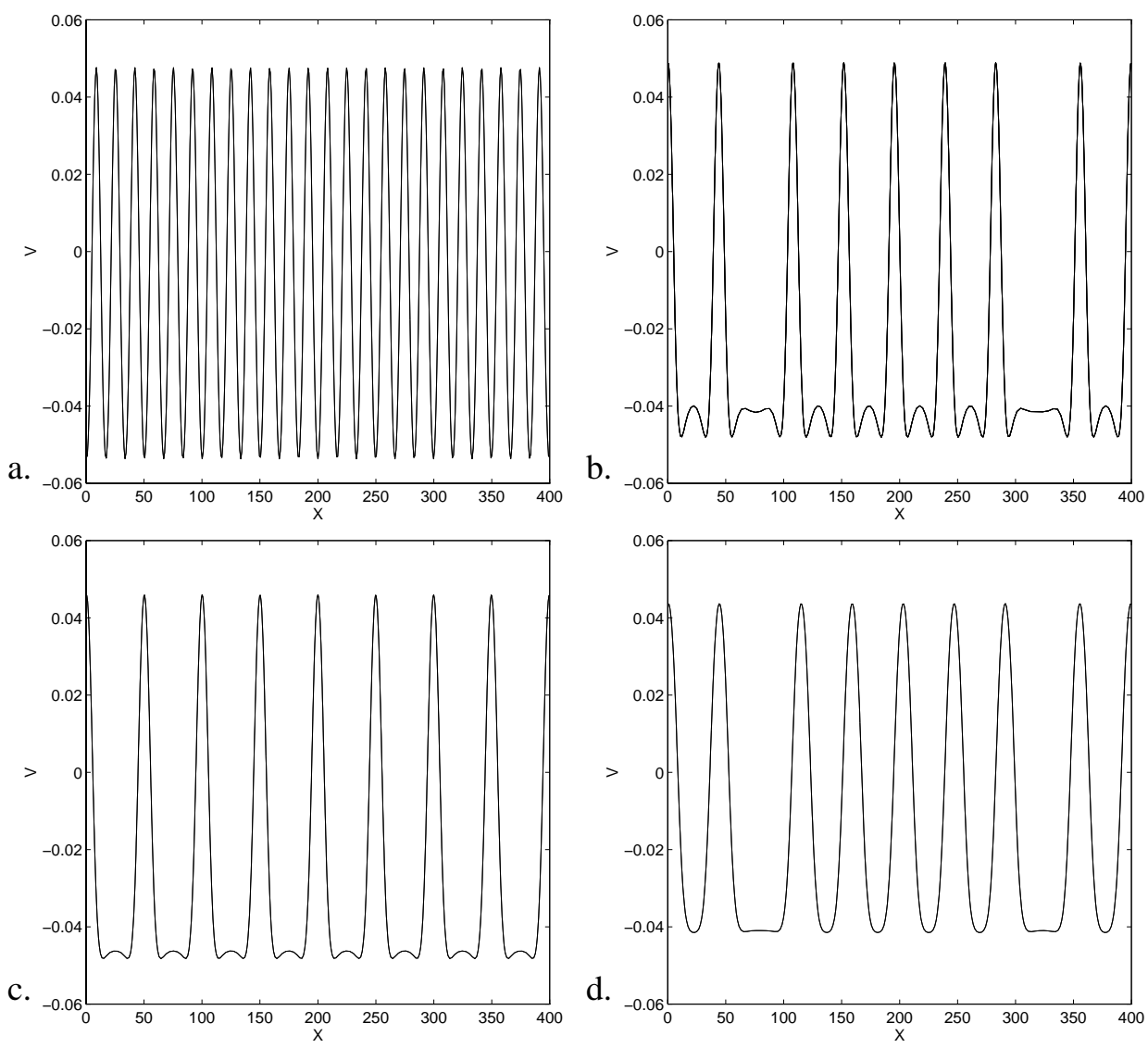

Figure 8: The $v$-component of the stable solutions for an initial condition that has 8 periods in the interval. The figures are given for the following choices of s: a. s=0.77 b. s=0.75 c. s=0.72 d. s=0.69 ( $N_{1}$ and $N_{2}$ are as in figure 7 ). 

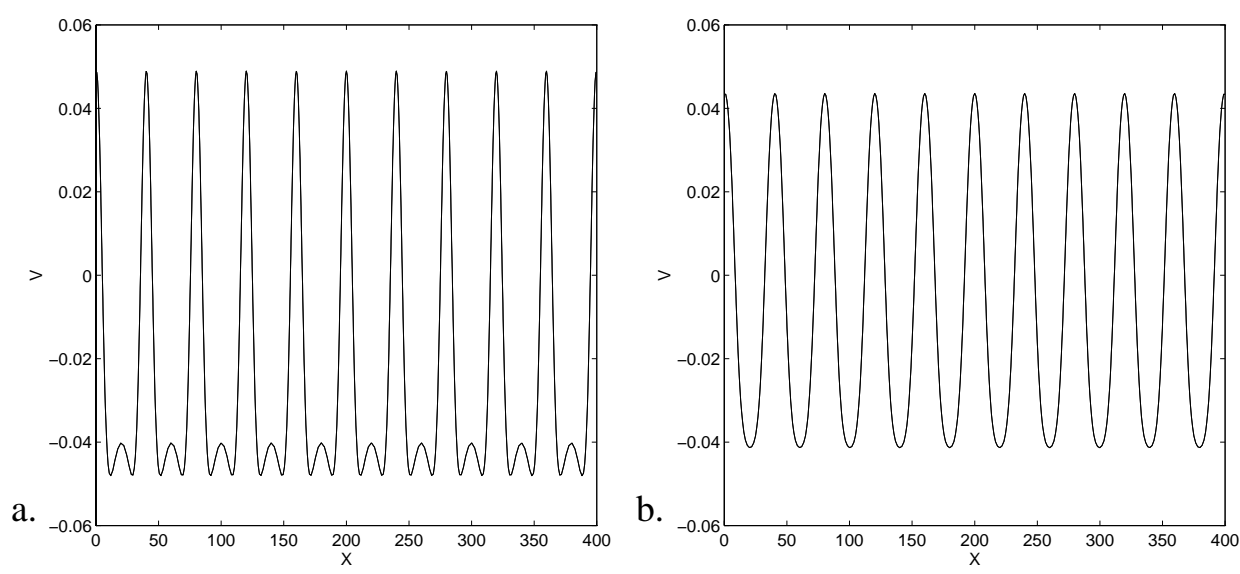

Figure 9: The $v$-component of the stable solutions with an initial condition that has 10 periods in the interval ( $N_{1}$ and $N_{2}$ are as in figure 7 ), a. $\mathrm{s}=0.75$ b. $s=0.69$
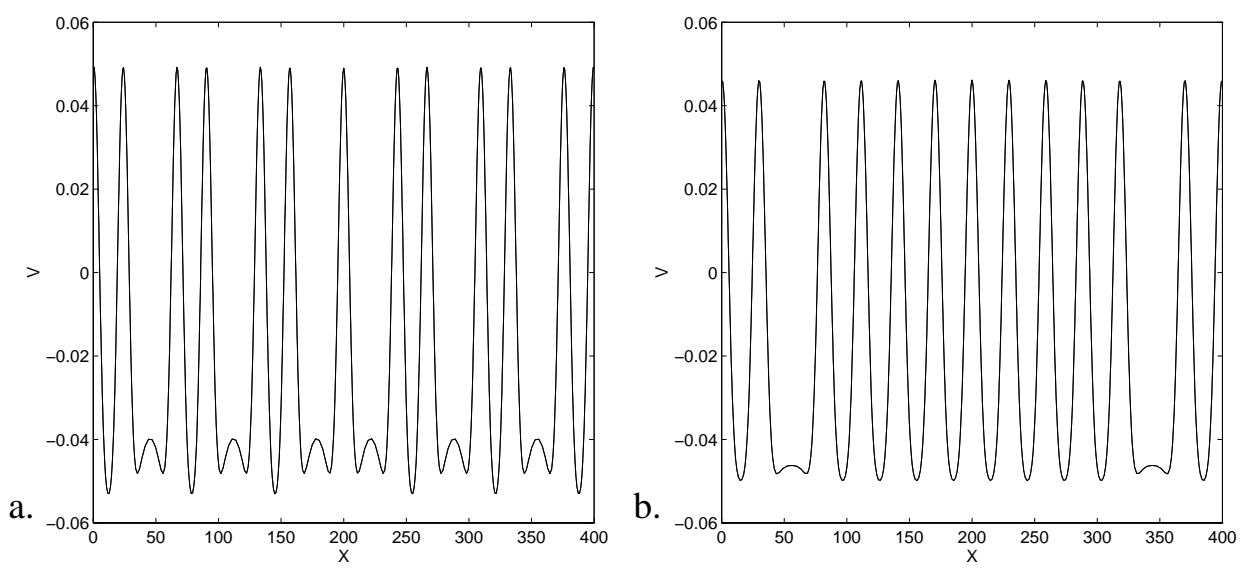

Figure 10: The $v$-component of the stable solutions with an initial condition that has 12 periods ( $N_{1}$ and $N_{2}$ are as in figure 7 ), a. $s=0.75 \mathrm{~b} . \mathrm{s}=0.72$ 

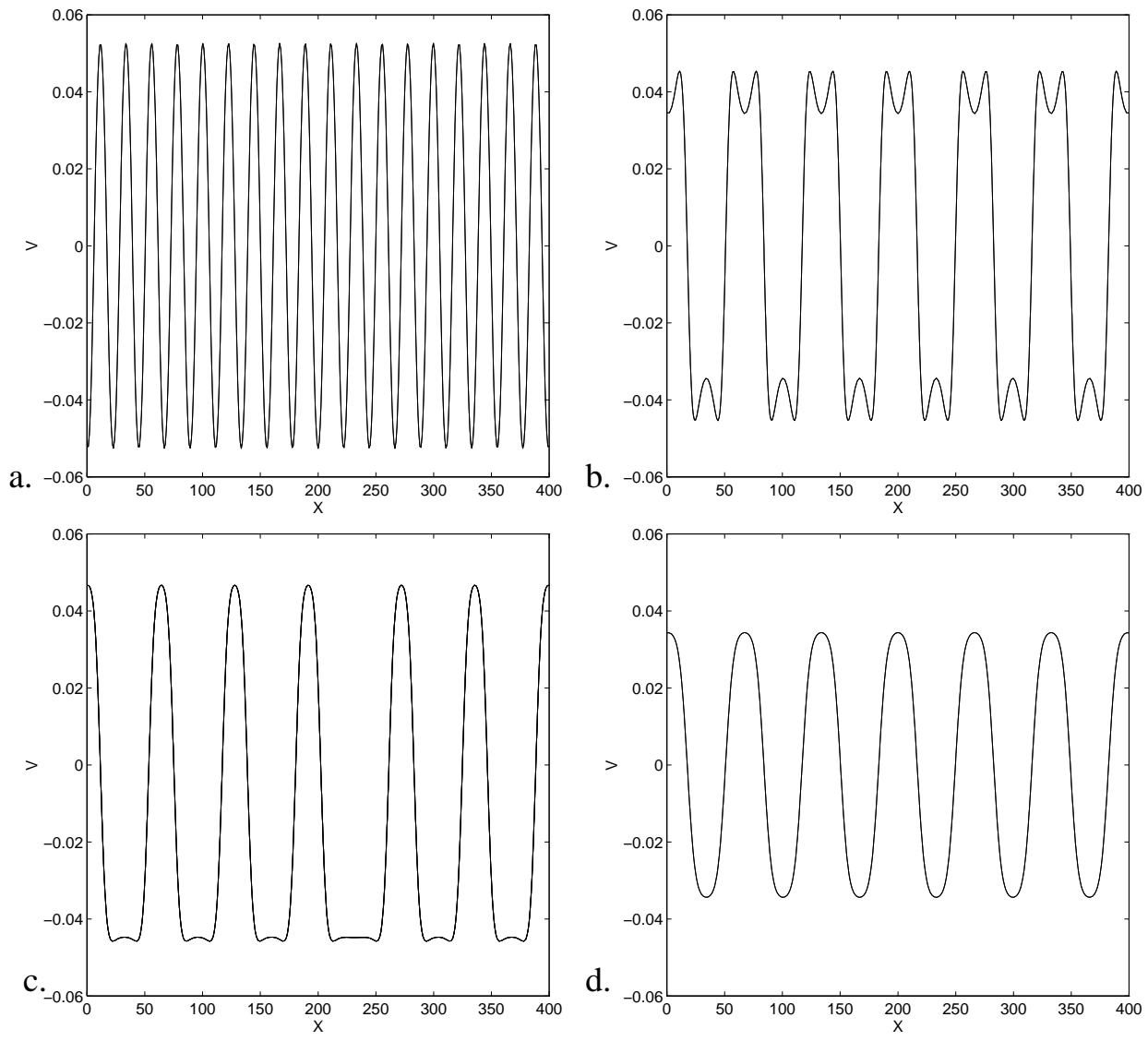

Figure 11: The $v$-component of the stable solutions with an initial condition that has 6 periods in the interval. Here $\alpha_{1}=\alpha_{2}=\alpha_{3}=\beta_{1}=\beta_{2}=\beta_{3}=$ $0, a=1$ and $b=3$ : the dynamics are described by the eFK3-equation. The figures are given for the following choices of $\mathrm{s}$ : a. $\mathrm{s}=0.76 \mathrm{~b} . \mathrm{s}=0.75 \mathrm{c} . \mathrm{s}=0.7$ d. $s=0.67$ 

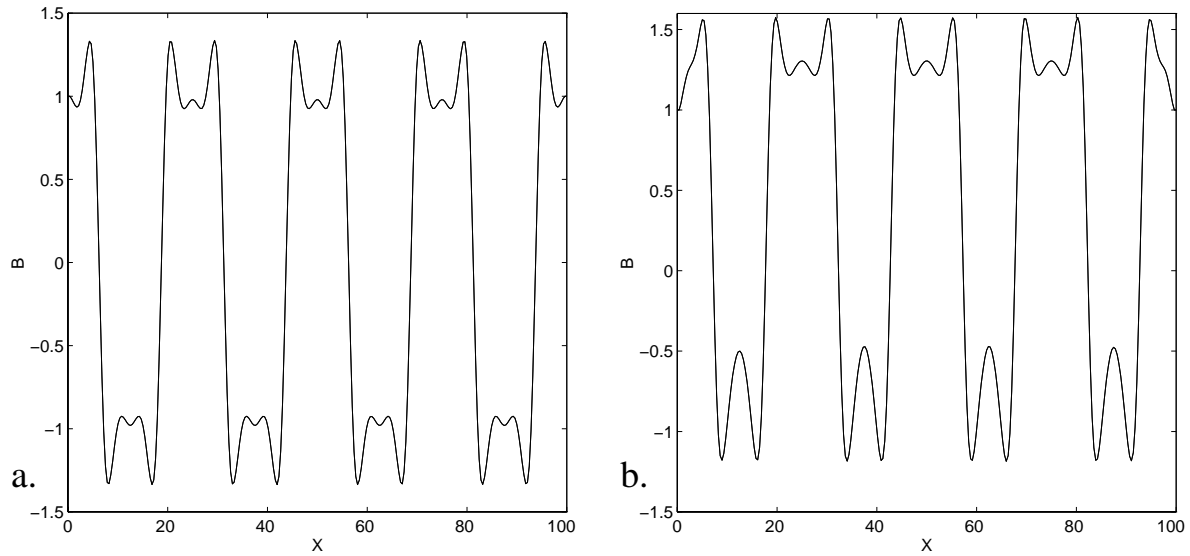

Figure 12: a. The stable solution for the eFK3-equation (3.27) with an initial condition that has 4 periods in the interval, here we chose $l=-1, D=$ -2 . b. The stable solution for the 'eFK2+3'-equation (5.1) with an initial condition that has 4 periods in the interval, here $D=-2, k=0.5$ and $l=-1$. 